Revue des patrimoines

$17 \mid 2011$

Les patrimoines de l'enseignement supérieur

\title{
Le Musée archéologique de la faculté des lettres de Bordeaux (1886)
}

L'institutionnalisation des collections pédagogiques et scientifiques

\section{Marion Lagrange et Florent Miane}

\section{OpenEdition}

\section{Journals}

Édition électronique

URL : http://journals.openedition.org/insitu/920

DOI : 10.4000/insitu.920

ISSN : $1630-7305$

Éditeur

Ministère de la culture

Référence électronique

Marion Lagrange et Florent Miane, « Le Musée archéologique de la faculté des lettres de Bordeaux

(1886) », In Situ [En ligne], 17 | 2011, mis en ligne le 29 novembre 2011, consulté le 20 avril 2019. URL

http://journals.openedition.org/insitu/920 ; DOI : 10.4000/insitu.920

Ce document a été généré automatiquement le 20 avril 2019

\section{cc) (1) $\odot$}

In Situ Revues des patrimoines est mis à disposition selon les termes de la licence Creative Commons Attribution - Pas d'Utilisation Commerciale - Pas de Modification 4.0 International. 


\title{
Le Musée archéologique de la faculté des lettres de Bordeaux (1886)
}

\author{
L'institutionnalisation des collections pédagogiques et scientifiques
}

\author{
Marion Lagrange et Florent Miane
}

« Les bâtiments du Musée étaient entourés de cours et de promenades plantées d'arbres. [...] En

dehors de ce bâtiment principal décrit par Strabon, il y avait des dépendances considérables, la bibliothèque, des salles de dissection où Hérophile et Érasistrate commencèrent les merveilles de l'anatomie ; des appareils d'astronomie [...]; des parcs où Philadelphe faisait

venir des animaux de toute espèce, un jardin d'acclimatation de plantes rares, enfin tout ce qui pouvait soutenir et encourager l'activité des

savants ${ }^{1}$. "

1 À l'image du mouseion dont le futur doyen de la faculté des lettres de Bordeaux, Auguste Couat, dresse l'historique et le fonctionnement, un nouveau modèle de développement scientifique voit le jour dans les années 1880 sur la base de la complémentarité entre collections et recherche. En 1886, soit quatre années après l'inauguration du Musée de sculpture comparée à Paris, le Musée archéologique de la faculté des lettres de Bordeaux est ainsi le premier à être créé dans un cadre universitaire français. Précédant ceux de Paris (1891) et de Lyon (1893), servant de référence à celui de Montpellier (1890), il accompagne la réforme de l'enseignement supérieur sous la Troisième république et l'introduction de l'archéologie et de l'histoire de l'art parmi les disciplines universitaires. Les moulages sont désormais associés à des photographies, des livres illustrés et des objets provenant de fouilles archéologiques. Cet ensemble étaye les fondements d'un enseignement prenant modèle sur l'université allemande, et ce dans la droite ligne des 
disciplines scientifiques où la collecte d'objets a renouvelé l'étude et la pédagogie dès le début du XIXe siècle.

Pourtant, ce Musée archéologique et ses collections sont peu connus ${ }^{2}$. Comme tant d'autres, ils furent relégués en raison du besoin accru d'espace et de la préférence accordée à d'autres supports pédagogiques. Le transfert de la faculté des Lettres sur le campus universitaire bordelais a entraîné en 1977 la mise en dépôt des collections au nouveau musée d'histoire régionale qui devait investir le bâtiment des facultés après de lourdes transformations ${ }^{3}$. Outre la perte de leur fonction pédagogique, les moulages d'antiques, qui constituent la part la plus visible du Musée archéologique, étaient dépossédés également de leurs locaux qui avaient été aménagés à dessein. Ils furent pour la plupart relégués dans des réserves. Néanmoins, à compter des années 1990, les recherches menées localement sur l'histoire de l'universitét, les études sur la genèse de l'enseignement universitaire en histoire de l'art ${ }^{5}$ et l'intérêt scientifique porté aux collections pédagogiques de moulages ${ }^{6}$ ont conduit à un premier travail sur la naissance de l'enseignement de l'histoire de l'art à la faculté des lettres'. Dans le cadre d'un programme de recherches "Région", une collaboration entre le musée d'Aquitaine et l'université de Bordeaux 3 a également permis des récolements ainsi qu'une étude des moulages et des photographies aujourd'hui existants 8 .

3 Sans s'attacher spécifiquement à la seule collection de moulages, la genèse de ce musée est ici abordée dans une perspective historique mettant en regard l'élaboration d'une pédagogie, la conception de nouveaux bâtiments et la construction d'une discipline universitaire. Il s'agit d'appréhender les interactions entre l'échelon local et national dans un contexte de réforme institutionnelle des facultés.

\section{Les premières collections scientifiques: des initiatives locales (1838-1862)}

4 Les premières collections scientifiques ont été constituées à Bordeaux peu après la fondation de la faculté de théologie, des sciences et des lettres par l'ordonnance du 24 août $1838^{\circ}$. Cette création fait partie d'un programme visant à améliorer l'enseignement supérieur dont le rapport Thénard (12 décembre 1837) a révélé les nombreuses faiblesses ${ }^{10}$. La réforme couvrant l'ensemble du territoire, l'État ne peut subvenir à tous les frais dont il a normalement la charge (loi du 17 novembre 1834). Les collections et les instruments scientifiques sont acquis par les municipalités qui financent également la construction et l'entretien des bâtiments (ordonnance du 17 février 1815).

Or la mairie de Bordeaux, n'ayant alors ni les locaux disponibles, ni les moyens de les bâtir, décide d'installer provisoirement les facultés dans une aile de l'hôtel de ville et dans les trois salles du musée de peinture qui lui sont contiguës. Un premier budget est affecté par le conseil municipal aux travaux de réaménagement de l'hôtel de ville (15 800 francs) ${ }^{11}$. Il permet d'établir, pour la faculté des sciences, un amphithéâtre, des laboratoires de physique et de chimie, des cabinets de physique, de géologie, de botanique, de zoologie et d'astronomie; pour la faculté des lettres, un amphithéâtre et trois salles de cours. Ces facultés regroupent sept chaires: littérature, philosophie (logique et métaphysique), histoire et géographie, calcul (intégral et différentiel), mécanique et astronomie, physique et chimie (théorique et expérimentale), histoire naturelle (géologie, botanique, zoologie) $)^{12}$. 
6 Un second budget est affecté à l'achat d'instruments, de collections et de livres (35000 francs) ${ }^{13}$. L'enseignement des lettres étant à cette époque uniquement théorique, seule la faculté des sciences est dotée de collections. Ces dernières se constituent parallèlement à celles des musées de la ville qui apparaissent comme autant de précieux auxiliaires. Alors que la municipalité aménage les locaux de la faculté pour recevoir des collections minéralogiques et zoologiques, elle achète la collection Roger pour le musée d'histoire naturelle. Les deux fonds viennent alors se compléter pour servir à l'instruction des étudiants ${ }^{14}$.

7 Les premières collections scientifiques de la faculté de Bordeaux ont donc été acquises entre 1839 et 1842 . Elles comprennent les collections de minéralogie, qui proviennent essentiellement de collectes et de dons ${ }^{15}$, les instruments de physique ${ }^{16}$, les collections de zoologie $^{17}$ et les instruments d'astronomie qui sont achetés par la ville ${ }^{18}$. Mais l'étroitesse des superficies dévolues aux laboratoires est rapidement réprouvée :

«[...] les amphithéâtres de MM. les professeurs se trouvent souvent inabordables par suite des exhalaisons délétères qui y arrivent par toutes les issues. Outre le danger qui en résulte pour la santé, une conséquence inévitable de cet état des choses est la prompte détérioration des instruments qui ne peuvent résister à l'action corrosive de l'atmosphère : déjà l'an dernier on a dû remplacer un de ces instruments qui était hors de service après deux années seulement. $M$. le professeur de chimie, ne pouvant continuer ses travaux dans le local actuel, se voit obligé de diviser ses opérations chimiques, il ne balance pas à louer à ses frais un petit local en dehors du bâtiment des facultés [... $]^{19}$ ».

8 Afin de remédier à ce double problème de l'espace et de l'organisation, $A_{b r i a^{20}}$, doyen de la faculté des sciences, réalise deux projets, l'un en 1858, l'autre en 1862, qui répondent aux besoins de la faculté tout en instaurant une nouvelle pédagogie. Le premier projet comprend la mise en place des services généraux (un amphithéatre de deux cent cinquante personnes et une bibliothèque de 10000 livres), d'un département de physique (un laboratoire pour les expériences et une galerie pour les collections), d'un département d'astronomie (une terrasse et un cabinet pour les instruments), d'un département de chimie (deux laboratoires pour les cours et pour le professeur, une salle pour les instruments mécaniques, une galerie pour les collections, un magasin pour les verreries et les produits, une salle pour la chaudière à vapeur) et enfin, d'un département d'histoire naturelle (un laboratoire d'anatomie, des galeries de géologie et de minéralogie, de botanique, de malacologie, d'ichtyologie, d'herpétologie, d'entomologie, d'ornithologie, de mammalogie, d'ostéologie, d'anatomie comparée) ${ }^{21}$. Le second projet complète le premier. Les capacités de l'amphithéâtre et de la bibliothèque sont doublées tandis que les salles de physique et de chimie sont augmentées d'un hangar pour réaliser les expériences, la terrasse astronomique est couverte d'une coupole métallique, l'histoire naturelle se dote d'un aquarium. Les galeries forment un musée ouvert à tous. Une annexe est affectée à la formation continue : géométrie descriptive, mécanique appliquée, physique appliquée, chimie appliquée, histoire naturelle appliquée ${ }^{22}$.

9 Une nouvelle conception de l'enseignement supérieur se dessine. Elle est notamment fondée sur une nouvelle gestion du savoir visible dans la répartition des espaces qui mêle à égale proportion les connaissances théoriques et l'expérience pratique. La faculté devient accessible prioritairement aux étudiants et aux chercheurs puisque seuls les amphithéâtres sont ouverts au grand public. Désormais, les laboratoires servent conjointement à la recherche et aux travaux pratiques et les collections scientifiques forment de véritables musées qui rivalisent avec ceux de la ville. Parallèlement, des cours 
pour adultes permettent à chacun de suivre l'évolution des techniques et des sciences et d'en profiter. La ville peut ainsi tirer tous les bénéfices de son engagement vis-à-vis des facultés comme cela a été défini dès l'origine :

«[La jeunesse bordelaise] pourra développer cette intelligence qui lui est si naturelle, et acquérir les connaissances que l'état actuel de la société rend nécessaires au commerçant, à l'artiste, à l'industriel et même au propriétaire ${ }^{23}$. "

Cependant, l'absence du soutien de l'État conduit à l'abandon des projets. L'université de France (Sorbonne) et les grandes écoles parisiennes semblent suffire aux besoins et le développement de l'enseignement supérieur en province n'apparaît pas comme une priorité. Les facultés provinciales restent un lieu ouvert à tout public, sans espace pour les collections et les expériences, où les livres s'entassent dans des bibliothèques trop étroites. L'enseignement supérieur, attirant essentiellement un public oisif et fortuné séduit par les nombreux cours du soir, relève encore très largement de la mondanité. Dans les années 1840, une quinzaine d'élèves y passe le baccalauréat chaque année. Ceux qui souhaitent poursuivre leurs études partent à Paris où ils s'inscrivent majoritairement en médecine et en droit $^{24}$. Si ces premiers projets restent sans suite, ils n'en constituent pas moins un modèle d'organisation de l'espace et de construction du savoir qui sera repris lors de la création du Musée archéologique de Bordeaux. Ces projets mêlent l'érudition (les cours), l'observation (les collections) et l'expérimentation (les travaux pratiques).

\section{Un nouveau bâtiment pour les facultés : le soutien de l'État (1875-1886)}

11 Le profond bouleversement social et politique qu'entraîne la défaite française de 1870 conduit à une réforme de l'enseignement supérieur. D'une part, l'État constate la faillite $\mathrm{du}$ modèle éducatif français, notamment celui des grandes écoles et du système centralisé; d'autre part, il s'agit de remplacer l'université de Strasbourg, devenue allemande après l'annexion de l'Alsace et d'une partie de la Lorraine. Ces constats remettent au goût du jour des observations déjà anciennes concernant la décentralisation des universités et le contenu des enseignements ${ }^{25}$. Il s'agit de multiplier les centres d'enseignement supérieur sur le territoire et de recourir davantage à l'expérimentation et aux travaux pratiques. Les universités deviennent alors un élément de cohésion nationale et de progrès technique. Elles paraissent pouvoir apporter les mêmes richesses qui ont profité à l'Allemagne lors de la chute du Premier Empire ${ }^{26}$.

12 À Bordeaux, l'idée d'installer la faculté dans des murs qui lui soient propres est relancée dès 1871 par Azam, professeur à la faculté de médecine ${ }^{27}$. Séduit par le prestige des universités d'Oxford, de Cambridge, de Leyde et d'Heidelberg, qui contraste avec la méconnue Université de France, il propose la création d'universités en province sur le modèle anglo-saxon et allemand tout en faisant du laboratoire la pièce maîtresse des facultés. L'originalité de son projet tient au fait qu'il se rattache au modèle américain (financement par des capitaux privés et mise en concurrence des centres universitaires). Il faut cependant attendre la dépêche du 6 février 1875 pour que l'État fasse savoir au conseil municipal son désir de faire de Bordeaux le siège d'une université complète ${ }^{28}$. Les facultés de théologie, de droit, de médecine, des sciences et des lettres seront réunies sous une même autorité, chacune ayant leur propre conseil. Assurée de l'aide de l'État, la ville de Bordeaux peut alors véritablement s'engager dans la construction d'un vaste 
bâtiment destiné à accueillir les facultés de théologie, des sciences et des lettres. En 1875, ce projet d'installation dans un nouveau bâtiment rue Vital-Carles est réalisé par l'architecte municipal Charles Burguet ${ }^{29}$. Un devis est fixé à 1650000 francs en fonction de la superficie du bâtiment et du coût des vitrines destinées à l'exposition des collections ${ }^{30}$. Il reste sans suite.

C'est finalement à Gaston Lespiault ${ }^{31}$ que revient le mérite d'avoir rendu possible la construction d'un bâtiment monumental (fig. $\mathbf{n}^{\circ} \mathbf{1}$ ). Professeur d'astronomie à la faculté de Bordeaux, il est aussi membre du conseil municipal. Cette double culture, scientifique et politique, lui permet d'inscrire les exigences de l'enseignement et de la recherche universitaire dans une vision globale des transformations urbaines. Il fait ainsi passer le devis des travaux dans l'emprunt de 6600000 francs destiné à moderniser la ville ${ }^{32}$. Dans son rapport, il évoque de nouveau le modèle allemand afin de convaincre le conseil municipal :

«[...] il faut à un établissement scientifique des bibliothèques, des collections, de vastes laboratoires, [...] irai-je encore prendre des exemples en Allemagne? Vous parlerais-je du palais universitaire de Berlin, qui fait le pendant du palais impérial ; des millions jetés par vingtaines dans les laboratoires de cette ville, dans ceux de Bonn et, faut-il le dire, dans ceux de Strasbourg?»

Figure 1

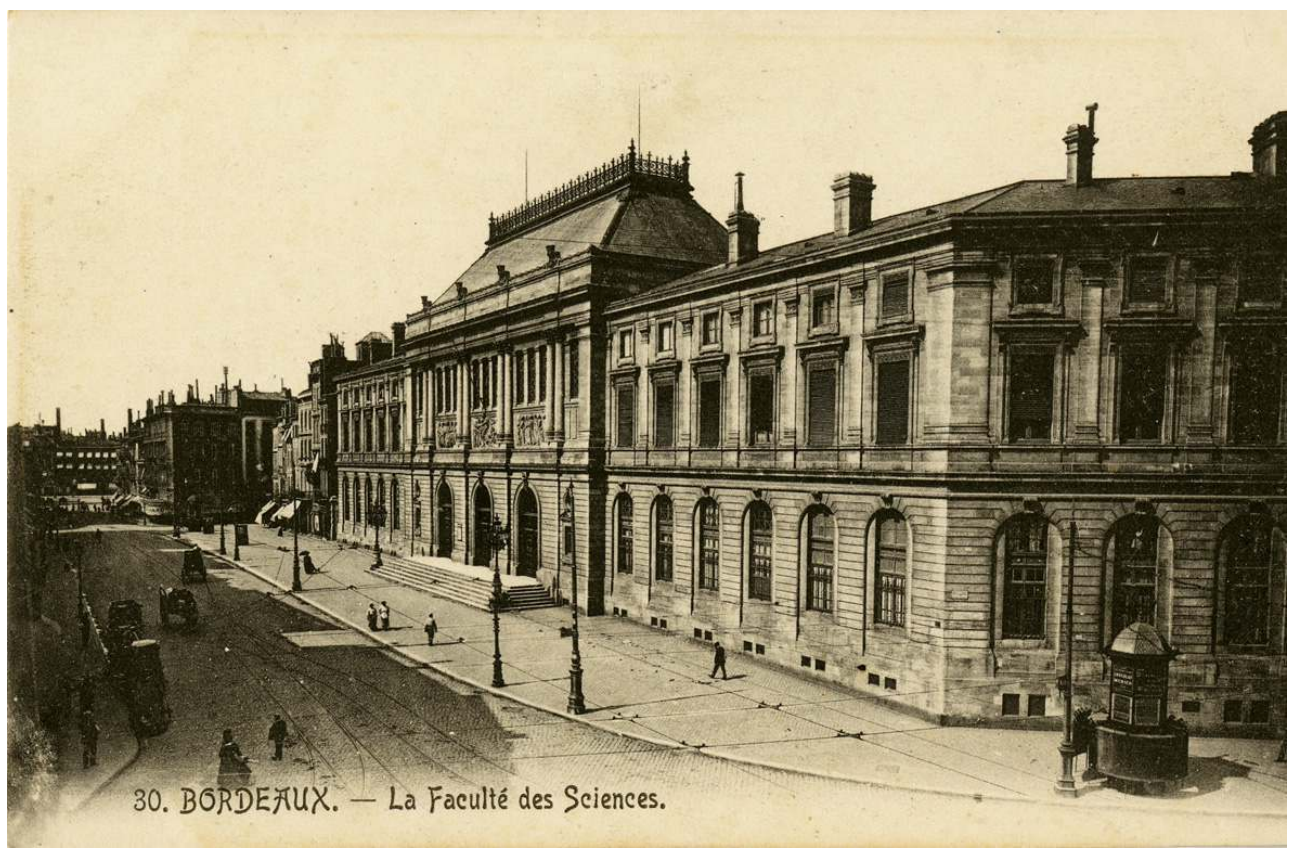

Anonyme. Faculté des sciences et des lettres de Bordeaux, ca 1900, carte postale, 9 × 13,8 cm Collection privée.

14 À la suite du rapport, le conseil municipal attribue 1500000 francs, à l'intérieur de l'emprunt, pour la construction des facultés. L'État, de son côté, s'est engagé à prendre en charge les collections et les instruments et a voté la création de trois cents bourses que vient compléter la création de six bourses par le département. Précédemment, le recteur de l'Académie de Bordeaux avait attiré l'attention du conseil sur l'utilitée ${ }^{33}$ et la rentabilité ${ }^{34}$ d'une telle affaire. Dans son rapport, Lespiault reprend l'idée d'une faculté destinée aux étudiants et aux chercheurs, équipée de bibliothèques, de collections et de laboratoires. Il 
rend compte de la création des chaires de littérature grecque, de géographie et d'archéologie qui apparaissent pour la première fois à Bordeaux.

De son côté, le doyen Roux ${ }^{35}$ fait parvenir au maire de Bordeaux un courrier daté du 31 mars 1879 indiquant les besoins de la faculté des lettres ${ }^{36}$. Il souligne que l'enseignement des lettres s'est complexifié et qu'il ne peut plus se contenter d'un petit amphithéâtre et de quelques salles de cours. Roux souhaite disposer d'un amphithéâtre de six cents personnes, de trois salles de cours et trois salles d'examens, de deux musées pour les collections de géographie et les collections archéologiques. Ces nouveaux espaces, qui apparaissent pour la première fois dans les documents officiels, montrent que l'enseignement des lettres prend modèle sur celui des sciences. Tendant à se confondre sur la forme alors que les locaux accueillent deux domaines de connaissance distincts, la surface octroyée aux sciences demeure toujours bien plus importante.

Alors que la lettre du doyen reflète des exigences des différents professeurs de lettres, l'idée d'un espace exclusivement consacré aux collections archéologiques revient sans doute à Louis Liard ${ }^{37}$ et à Maxime Collignon ${ }^{38}$. Toutefois, Pierre Paris ${ }^{39}$ sera le véritable maitre d'ouvrage de ce musée qui renouvelle tout à la fois le rapport à l'objet, la méthode d'enseignement et l'approche scientifique.

\section{Le Musée archéologique, la matérialisation d'un enseignement}

17 Le 21 décembre 1886, un décret officialise la création du Musée archéologique à la faculté des lettres de Bordeaux. Le 17 janvier de la même année, le nouveau bâtiment avait été inauguré avec les honneurs dus à un édifice public financé en grande partie par la ville, avec le soutien de l'État. Outre René-Marie Goblet, ministre de l'Instruction publique, accompagné d'Edmond Turquet, sous-secrétaire d'État aux beaux-arts, était présent le directeur de l'enseignement supérieur, Louis Liard, l'artisan de la réforme institutionnelle de l'université ${ }^{40}$. Il avait été professeur de philosophie au sein de la faculté bordelaise (1874-1880) et également adjoint au maire, délégué à l'instruction publique de la ville de Bordeaux $^{41}$. La construction ex-nihilo d'un vaste édifice pour accueillir les facultés des lettres et des sciences avait conduit Liard à mettre en œuvre un programme attestant d'une nouvelle conception de l'enseignement universitaire :

« À ma connaissance - écrit-il à l'architecte municipal Charles Durand - il n'est en France aucun édifice que vous puissiez prendre pour modèle. Les plans de nos anciennes Facultés des Sciences et des Lettres, dérivent sans exception, d'une conception de l'Enseignement supérieur qui commence à disparaître. Jusqu'à ces dernières années, les leçons [...] s'adressaient exclusivement au grand public, mélange de savants et de lettrés, et de curieux et d'oisifs, sans cesse renouvelés. De là ces vastes amphithéâtres, ouverts à tout venant. De là aussi l'absence de laboratoires d'études; à peine quelques salles étroites de recherches pour les professeurs; peu ou pas de collections et de bibliothèques. Aujourd'hui [...] les efforts communs de l'administration centrale de l'Autorité académique, et des professeurs, tendent de plus en plus à grouper autour de chaque chaire de véritables étudiants. [...] Cela vous fournira l'idée directrice de votre œuvre. On l'a dit avec justesse, une faculté doit être désormais un atelier ; de là, pour les lettres, des salles de conférences intimes, pour les sciences des laboratoires de recherches pour les professeurs, des laboratoires d'études pour les étudiants, des salles de collections voisines des laboratoires, des salles de cours aussi nombreuses que les divers enseignements de la Faculté ; enfin une vaste bibliothèque ${ }^{42}$. " 
18

En revanche, le programme tel qu'il est énoncé n'accorde aux collections archéologiques qu'une place relativement dérisoire par rapport aux espaces dédiés aux laboratoires scientifiques. Liard envisage une salle, mais évoque surtout l'idée d'une répartition des moulages dans des espaces de circulation ${ }^{43}$. L'architecte concèdera finalement une pièce de cent vingt mètres carrés placée au dernier niveau. Pourtant, un mois à peine après l'inauguration, Charles Durand se rend à Paris pour y rencontrer Louis Liard et Edmond Turquet en prévision notamment de la transformation des cours ouvertes de la faculté ${ }^{44}$. Peu de temps après, le 21 mai 1886, Auguste Couat ${ }^{45}$, tout à la fois doyen de la faculté des lettres et nouvel adjoint au maire chargé de l'instruction publique, soumet au conseil municipal un projet de Musée archéologique dans la cour de la faculté des lettres (fig. $\mathbf{n}^{\circ}$ 2). Suite au rapport qu'en fit la commission de l'Instruction publique de la ville, les frais de construction et d'aménagement "d'une des deux cours carrées pour y installer un musée de moulages comprenant photographies et reproductions archéologiques ${ }^{46}$ " sont intégralement pris en charge par la municipalité à hauteur de 12587,93 francs. Lors du discours solennel prononcé à l'occasion de la rentrée universitaire en novembre 1886, le recteur annonce que l'État participera à l'achat des moulages pour un total de 50000 francs $^{47}$.

Figure 2

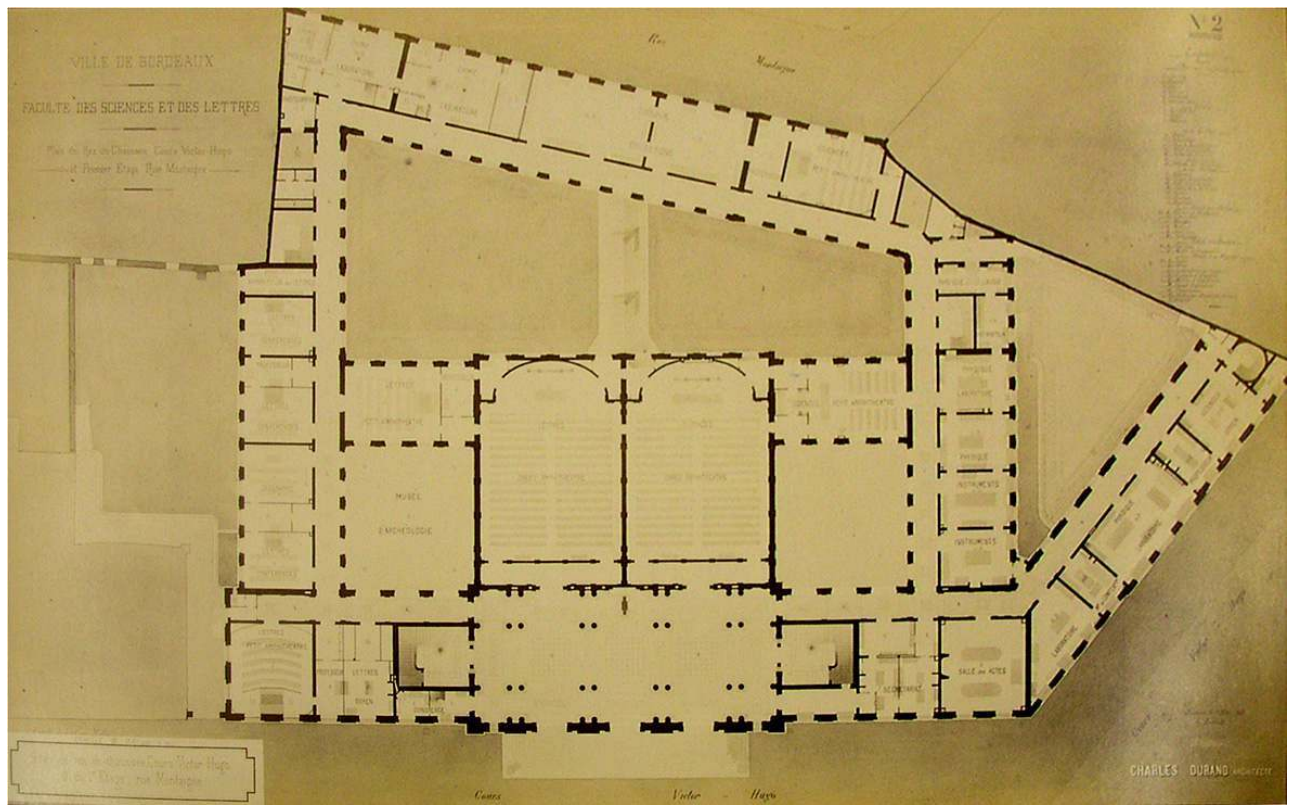

TERPEREAU, Jules-Alphonse. Plan de la faculté des sciences et des lettres par Charles Durand. ca 1886, papier albuminé collé sur bristol, $26 \times 41,8 \mathrm{~cm}$.

(c) Bibliothèque municipale de Bordeaux.

Si cet aménagement bordelais joue à ce moment-là le rôle de précurseur et de référent à la fois pour les facultés de province ${ }^{48}$ et pour la Sorbonne, la création des premières chaires d'archéologie au milieu des années 1870 avait déjà été suivie par des achats de moulages et, avec eux, d'une réflexion sur le lien entre l'enseignement et la recherche. La nomination de Maxime Collignon (1849-1917) à Bordeaux, comme chargé de cours sur une chaire d'antiquités grecques et latines créée par décret ministériel dès le 31 octobre 1876 ${ }^{49}$, eut indéniablement un rôle moteur dans la fondation de la méthodologie universitaire. Agrégé de lettres, ancien membre de l'École française d'Athènes (1873-1876) et de l'École 
française de Rome (1873-1874), il est considéré comme l'un de ceux qui « ont le plus accru et popularisé les explorations et les découvertes archéologiques ${ }^{50}$ ». Lors de sa leçon d'ouverture le 15 janvier 1877, il célèbre l'archéologie comme une science qui «prend place dans l'enseignement supérieur, non pas à titre d'auxiliaire, mais avec ses méthodes propres et ses caractères originaux ${ }^{51} \%$.

20 S'appuyant sur son expérience de fouilles en Italie et en Grèce, Collignon « a fait l'histoire de la sculpture en Grèce, depuis la période archaïque, jusqu'à l'école de Phidias. [...] [II] a étudié, d'après les moulages, la décoration sculpturale du Parthénon ${ }^{52} »$. Son enseignement d'une "histoire de l'art antique ", ainsi qu'il la nomme, repose dès les premières années bordelaises sur l'étude de premiers moulages acquis sans nul doute à sa demande. Il est par ailleurs chargé trois ans plus tard, par le ministre de l'Instruction publique, d'un rapport portant notamment sur la place en Allemagne des collections archéologiques dans l'enseignement ${ }^{53}$. Ce travail offre l'occasion de montrer, outre leur avancée en la matière, que les ensembles de moulages dans un cadre universitaire doivent se distinguer des prestigieux exemples - comme le Museum der Gypsabgüsse de Dresde qui les ont précédés :

«Ces collections n'ont pas été créées sans difficulté, et la bonne volonté des professeurs s'est souvent heurtée au mauvais vouloir ou à l'indifférence. [...] Le résultat de tous ces efforts, c'est qu'aujourd'hui le principe est reconnu sans contestation. Des sommes plus ou moins importantes sont affectées à l'entretien des collections [...] sous le nom d'apparat archéologique. Ce titre désigne d'une façon générale tout le matériel qui sert à l'enseignement : moulages en plâtre, empreintes de médailles ou de pierres gravées, Vorlegeblätter, etc.; on y comprend aussi les livres usuels [...] qui forment une bibliothèque spéciale, distincte de la Bibliothèque universitaire ${ }^{54}$. »

21 Au-delà de cet éventail d'objets à la disposition des étudiants, Collignon insiste sur la nécessité d'agencer le musée par rapport à un discours historique :

"Pour rendre tous les services qu'on peut en attendre, un musée de moulages doit offrir une série de monuments méthodiquement classée dans l'ordre chronologique, comprenant les types les plus caractéristiques, ouverte aux accroissements que rendent nécessaires les découvertes nouvelles. Il faut qu'une visite attentive soit à elle seule un enseignement, et que le visiteur en emporte une idée très nette du développement historique de l'art ${ }^{55}$. "

Collignon se démarque alors par la volonté de faire converger une vision historique des objets antiques et la réalité matérielle des œuvres ${ }^{56}$. L'enseignement généraliste s'accompagne de cours spécialisés sur les récentes découvertes (statuettes de Tanagra) et sur des domaines peu étudiés (céramiques grecques) tandis que le discours scientifique s'appuie sur des outils didactiques ${ }^{57}$. En effet, une méthode d'organisation et une classification nécessaires à l'agencement d'une collection de moulages, dont le catalogue est généralement la matérialisation, servent les fondements scientifiques de la discipline universitaire. Celle-ci doit aussi prendre ses distances avec la seule érudition. Près de vingt ans après la parution de son rapport, Collignon se félicite «l'idée d'une galerie de moulages est le complément nécessaire des chaires où l'on enseigne l'histoire de l'art antique [...] [et] elle n'a pas besoin d'être justifiée par une démonstration qui n'est plus à faire ${ }^{58} »$.

23 Le jeune professeur quitte la faculté des lettres de Bordeaux en 1883 pour devenir suppléant de Georges Perrot à la Sorbonne. Il n'aura pas eu le temps de voir s'achever le chantier de transfert des facultés initié par Louis Liard - devenu en 1880 recteur de l'Académie de Caen - et, surtout, se matérialiser un musée archéologique tel qu'il l'avait 
conceptualisé et tel qu'il le mettra en œuvre seulement quelques années plus tard dans les nouveaux bâtiments de la Sorbonne ${ }^{59}$. Sa chaire est reprise entre 1883 et 1884 par un suppléant, Bernard Haussoullier (1853-1926) ${ }^{60}$, agrégé de lettres et ancien membre de l'École française d'Athènes (1875-1876). Camille Jullian (1859-1933), agrégé d'histoire et de géographie, ancien membre de l'École française de Rome (1880-1882), épigraphiste de formation, lui succédera à partir de 1885 après avoir été chargé durant deux ans d'un cours complémentaire d'histoire ancienne et d'antiquités grecques et latines. Son enseignement s'articulera autour de l'histoire de Bordeaux et du sud-ouest à partir de l'étude des inscriptions, des monuments et de la topographie ${ }^{61}$. Au même moment, Pierre Paris (1859-1931), normalien, ancien membre de l'École française d'Athènes (1882-1885), est chargé d'un cours complémentaire de langues et littérature grecques (arrêté du 13 novembre 1885) (fig. $\mathbf{n}^{\circ} \mathbf{3}$ ). Suite à sa soutenance de doctorat de lettres, il devient, à la rentrée de novembre 1886, maitre de conférences (arrêté du 7 août 1886) ${ }^{62}$ d'une chaire en archéologie et institutions grecques jusque-là inexistante à la faculté des lettres de Bordeaux. Ce jeune homme, qui débute sa carrière universitaire, reprend l'héritage laissé par son éminent prédécesseur : responsable de l'aménagement du musée archéologique et de son enrichissement, il partage avec Collignon une vision de l'archéologie comme système narratif devant s'appuyer sur l'étude des objets ainsi qu'un enseignement basé sur la pédagogie et la contribution des étudiants.

Figure 3

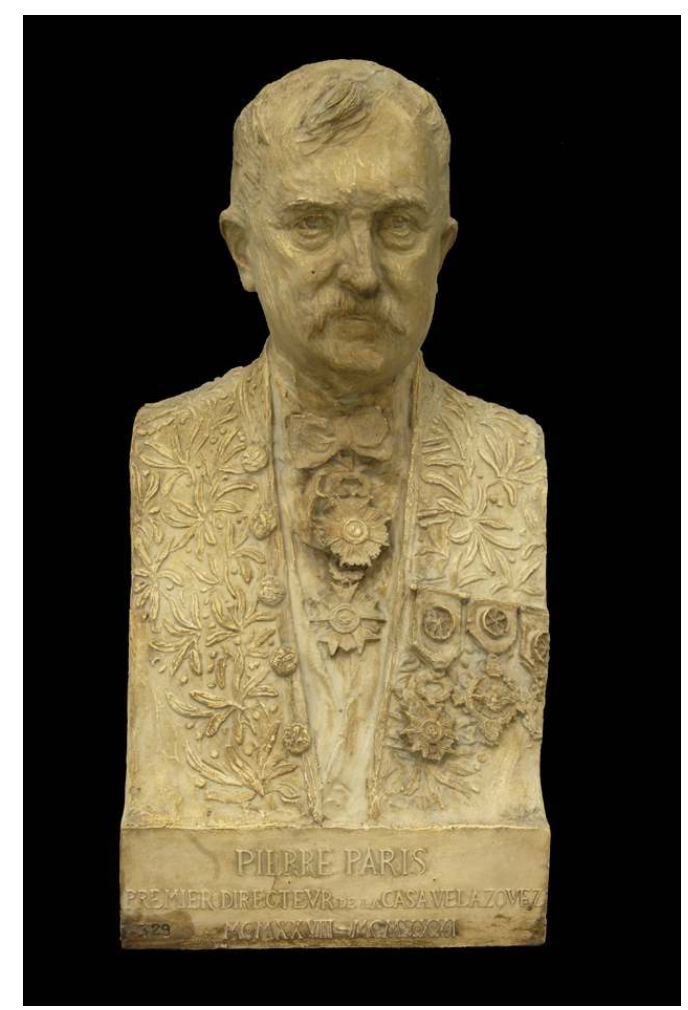

Mariano Benlliure. Buste de Pierre Paris. 1932, plâtre peint.

Phot. Armand Neble. (c) Université de Bordeaux 3.

Lors de la rentrée solennelle des facultés en novembre 1886, le doyen Auguste Couat confie officiellement à Pierre Paris les rênes de cette institution : 
«[...] la Faculté ne disposait que d'un nombre insuffisant de moulages; il était d'ailleurs impossible d'en réunir davantage dans l'ancienne Faculté. Une vaste salle avait été réservée dans la Faculté nouvelle pour notre collection. Cette salle a été trouvée trop petite ; on s'est dit qu'un monument aussi vaste et aussi beau pouvait servir à former un musée d'antiques, unique peut-être dans les Facultés de province. Une des cours qui avoisinent les grands amphithéâtres va être vitrée et transformée en un musée plus petit mais analogue à celui de la grande cour vitrée de l'École des Beaux-Arts de Paris. La ville, inépuisable dans ses largesses, a bien voulu donner les fonds pour l'installation de la cour ; l'État fournira les moulages. Notre collègue, M. Pâris [sic], pourra se féliciter d'avoir à sa disposition, pour ses études personnelles et pour son cours, des richesses qu'il ne trouverait, sans doute, nulle part ailleurs ${ }^{63}$."

Si le doyen manifeste en premier lieu son attachement à la dimension ostentatoire d'une collection de moulages d'antiques, destinée à être placée dans un lieu évoquant la prestigieuse institution parisienne, il rappelle également que le choix des sculptures, puis leur installation, doivent répondre à des exigences historiques et artistiques dans la mesure où cette collection est intimement liée à un enseignement (fig. $\mathbf{n}^{\circ}$ 4), (fig. $\mathbf{n}^{\circ} 5$ ) (fig. $\mathbf{n}^{\circ}$ ).

Figure 4

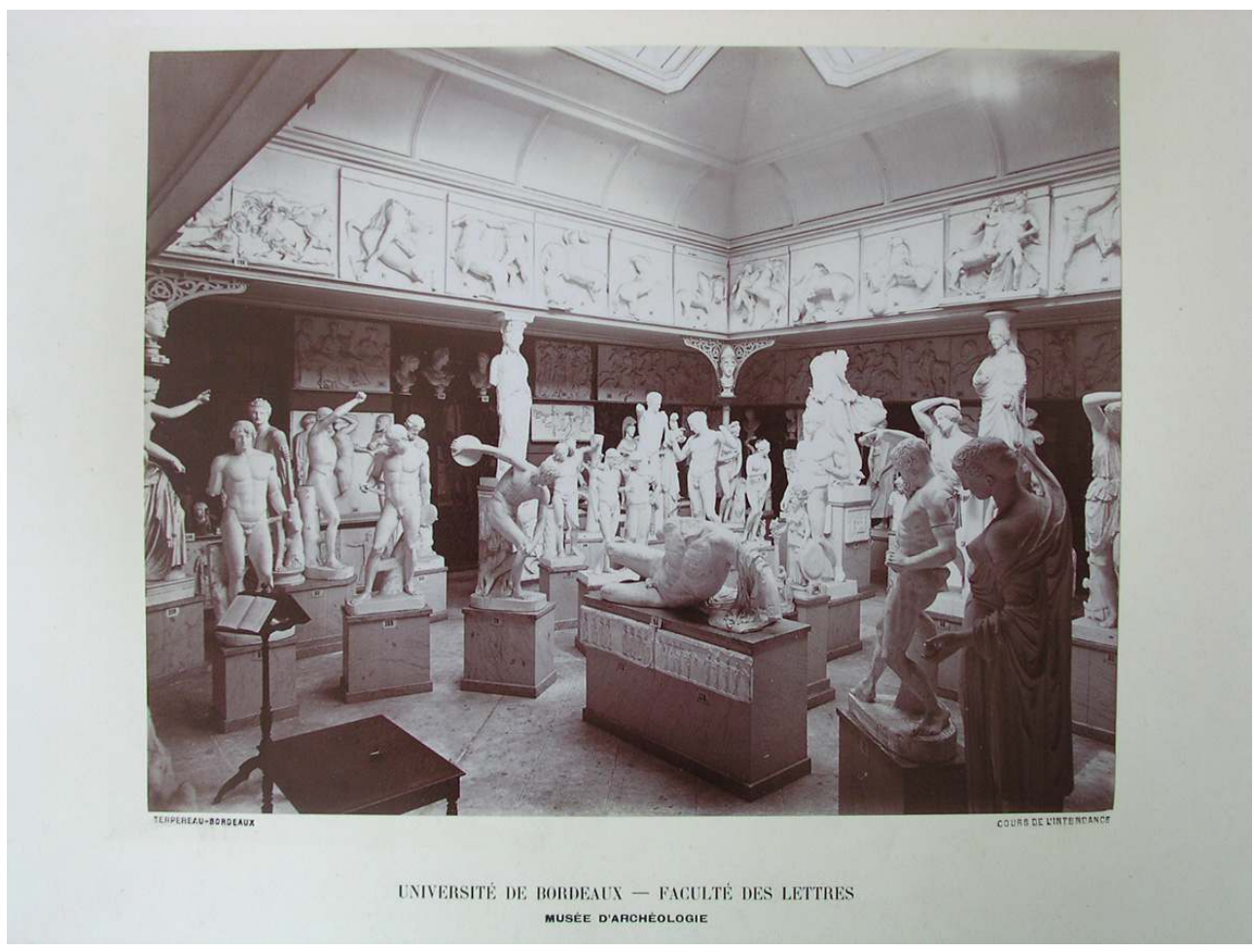

TERPEREAU, Jules-Alphonse. Musée archéologique de la faculté des lettres de Bordeaux. ca 1886, papier albuminé collé sur bristol, 19,5 × 24,8 cm.

(C) Université de Bordeaux 3 
Figure 5

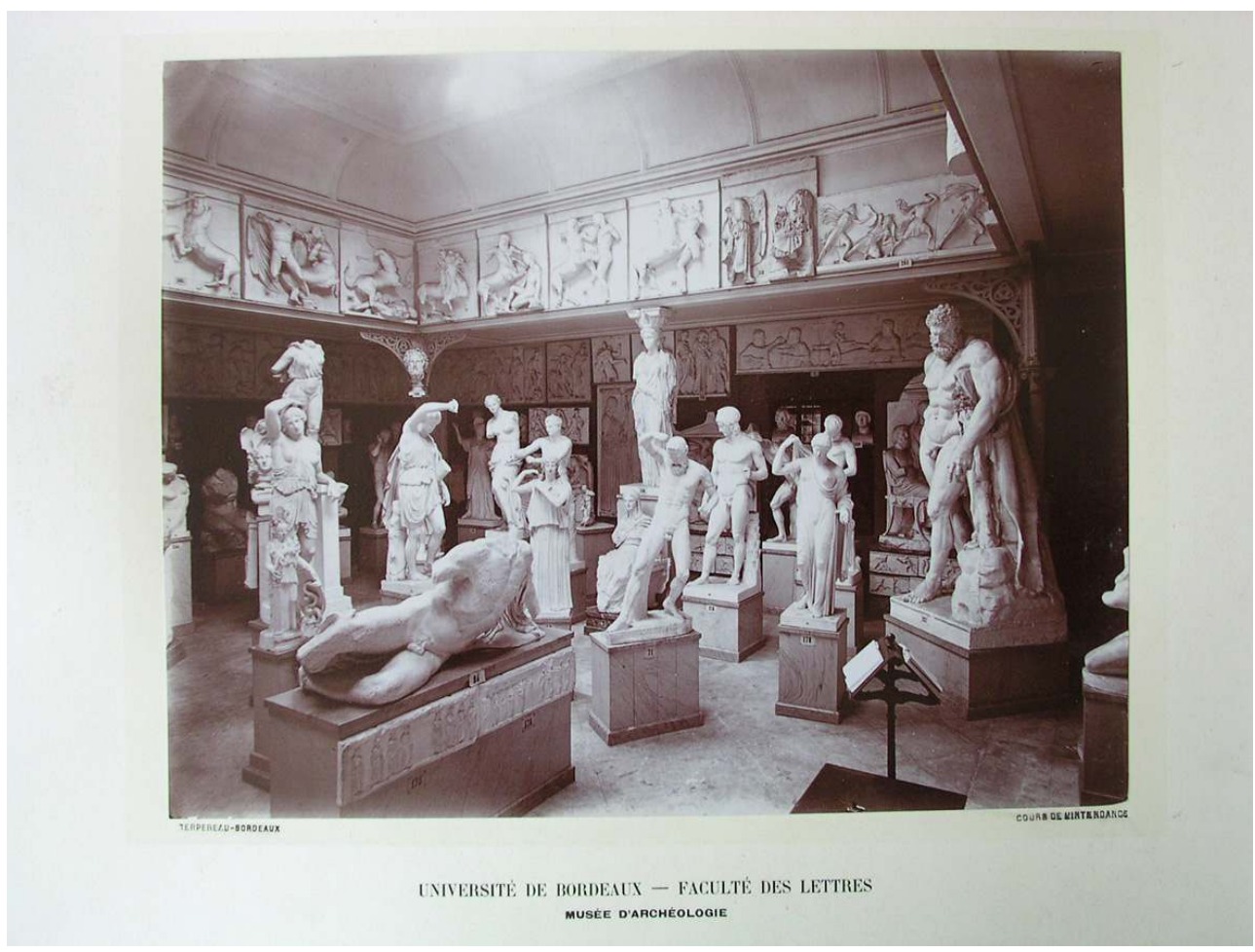

TERPEREAU, Jules-Alphonse. Musée archéologique de la faculté des lettres de Bordeaux. ca 1886, papier albuminé collé sur bristol, 19,4 × 24,9 cm.

(c) Université de Bordeaux 3. 


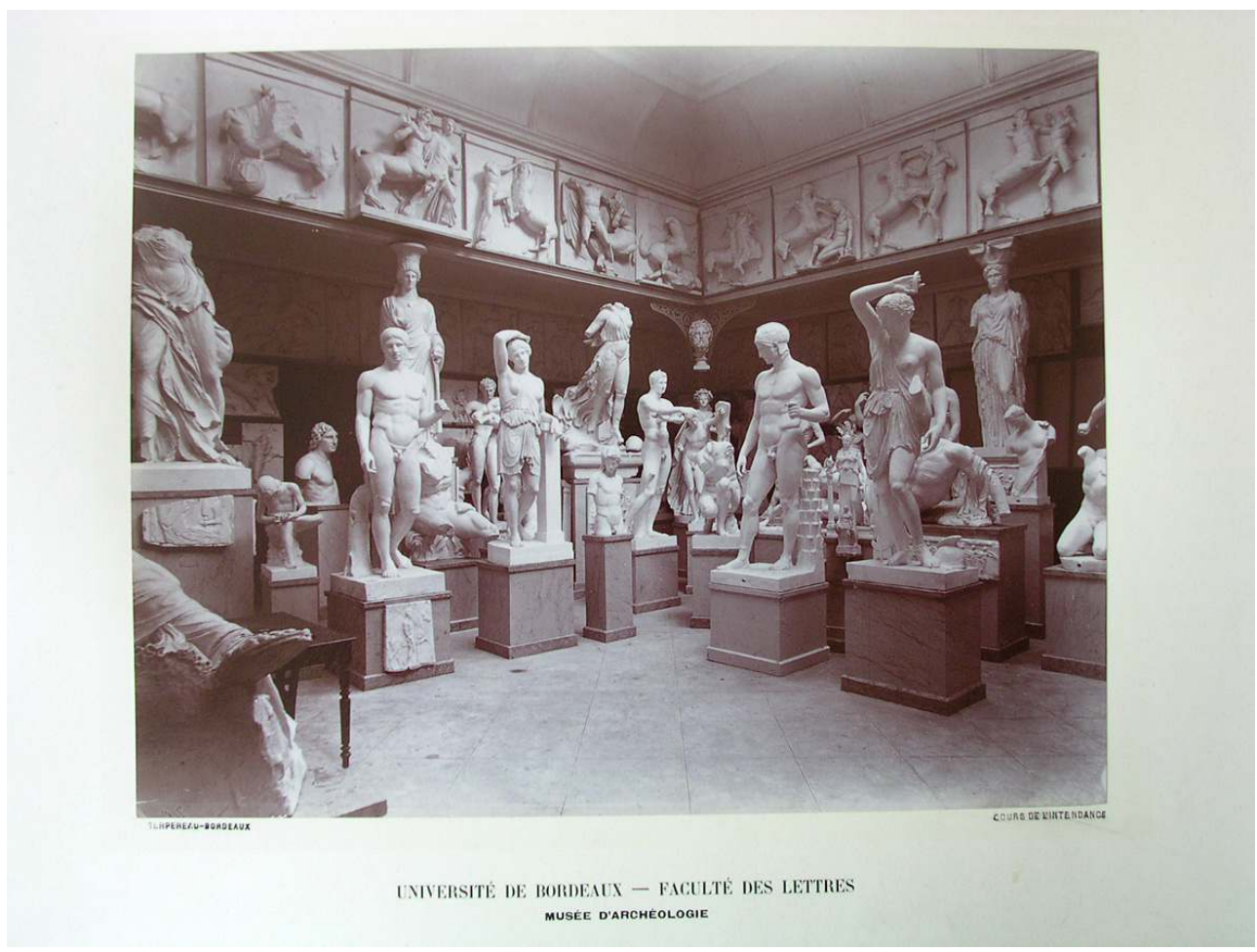

TERPEREAU, Jules-Alphonse. Musée archéologique de la faculté des lettres de Bordeaux. ca 1886, papier albuminé collé sur bristol, $19,4 \times 24,9 \mathrm{~cm}$.

(C) Université de Bordeaux 3.

L'année suivante, le nouveau doyen annonce que Pierre Paris a achevé «la laborieuse installation de ce musée archéologique ${ }^{64} »$. Mais la question de l'espace disponible ne tarde pas à revenir au premier plan :

«Pourquoi faut-il que cette collection à peine achevée soit déjà à l'étroit dans le local que la ville a bien voulu disposer pour la recevoir? La patience et le goût du professeur ont réussi à rendre le trop réel encombrement de notre salle presque agréable à l'œil ; mais que ferons-nous des nouveaux moulages qui sont encore attendus ? $?^{65}$,

Pourtant, l'effort prodigué par Paris se voit récompensé de manière numéraire par le directeur de l'enseignement supérieur, Louis Liard, qui lui délivre une indemnité extraordinaire de mille francs ${ }^{66}$. Parallèlement, le conseil des facultés concède à partir de 1887 un crédit d'une somme identique "pour l'installation, l'entretien du musée archéologique et les achats courants destinés à augmenter ses richesses ${ }^{67}$ ». Lorsque l'assesseur demande quelques mois plus tard une augmentation de cent cinquante francs, il lui est accordé aux dépens du crédit de 13000 francs des collections de la faculté des sciences ${ }^{68}$. Cette même année, un nouveau projet d'aménagement voit le jour dans la cour située à l'arrière des bâtiments des facultés des lettres et des sciences. Malgré l'examen des plans sur les lieux par le directeur de l'Enseignement supérieur ${ }^{69}$ et une convention signée entre les deux facultés ${ }^{70}$, le projet sera sans cesse repoussé. Trois ans plus tard, la question est en vain examinée en Conseil général des Facultés, impliquant un investissement de 30000 francs $^{71}$. Pourtant, en 1893, le projet semble relancé par le doyen Stapfer : 
«Monsieur le Recteur, [...] Après un commun accord entre MM. Les Doyens des deux Facultés conclu en présence de M. Liard, notre Directeur, ces salles et cette cour furent attribuées à la Faculté des Lettres pour y installer dans la cour, un vaste pavillon qui serait le musée d'Archéologie et dans les salles, des collections photographiques, une Bibliothèque spéciale et un Cabinet, dépendance du dit musée. Aujourd'hui, et en attendant la possibilité de réaliser ce projet nous avons besoin d'argent et besoin, vu l'encombrement, d'occuper deux des salles en question et d'y placer les moulages dont regorge le petit pavillon vitré actuel. J'ai donc l'honneur, Monsieur le Recteur, de vous prier de vouloir bien demander à $\mathrm{M}$. le Maire de faire achever ces salles ${ }^{72}$.» trois salles de l'entresol prévues pour être annexées au musée lorsque celui-ci s'installera dans la cour réservée. Toutefois, le professeur ne cache pas son désappointement :

«Mais ces trois salles sont malheureusement dans un état tout à fait indigne des œuvres qui y sont exposées. C'est qu'elles n'ont jamais été terminées : elles n'ont ni plancher ni dallage; les murs sont blancs et nus; les portes et les boiseries n'ont pas été posées. Au courant de l'année dernière, vous avez bien voulu faire connaître la situation à l'administration municipale et réclamer l'achèvement de ce local. Un devis a été dressé par les soins de l'architecte de la ville, mais les travaux, par suite de circonstances que j'ignore, n'ont pas été entrepris ${ }^{73}$. »

Il faut attendre encore un an pour que l'aménagement soit réellement voté en conseil municipal avec un crédit supplémentaire de 2300 francs :

«Sans doute, ces salles ne seront jamais très luxueuses, mais elles permettront, du moins, de dégager le Musée actuel. On y réunira les œuvres archaïques, non pas les moins intéressantes, mais celles qui demandent, pour être appréciées, une éducation préalable; les étudiants y travailleront à leur aise et rien ne s'opposera plus à ce que le public puisse pénétrer dans le Musée ${ }^{74}$. "

Désormais, la collection de moulages se répartira entre la salle du musée proprement dite, avec les chefs-d'œuvre de la sculpture grecque et romaine, la salle anciennement attribuée aux collections archéologiques, avec le double fronton du temple de Zeus à Olympie ${ }^{75}$, et les trois salles, deux étant désormais consacrées à l'art archaïque ${ }^{76}$. Ces objets ordonnancés par période apparaissent d'abord comme un complément naturel au cours public professé par Paris sur la sculpture grecque classique à partir de $1887^{77}$. Les enseignements sont réalisés dans l'enceinte même du musée et tous les étudiants y sont $\mathrm{admis}^{78}$. En outre, les élèves de l'école municipale des beaux-arts sont autorisés à venir y dessiner. Mais cette collection de moulages est également un outil didactique sur lequel s'appuie le professeur pour faire travailler ses étudiants dans le cadre d'exercices pratiques d'archéologie.

31 Reprenant un mode opératoire désormais en usage dans le cadre des musées, Pierre Paris accompagne ses étudiants dans la rédaction d'un catalogue dont la publication est autorisée par le doyen Espinas ${ }^{79}$ et le recteur Ouvré dès le 12 décembre $1888^{80}$. Paru dans un premier temps sous forme de deux fascicules entre 1889 et 1890, l'ouvrage est édité en 1892 sous le titre de Catalogue méthodique des moulages des cuvres de sculpture grecque. Classé par périodes, par école et par style, chacun des deux cent quatre-vingt numéros (représentant plus de trois cent cinquante moulages) ${ }^{81}$ fait l'objet d'une notice. Toutes les sculptures sont replacées dans leur contexte de découverte (date et lieu des fouilles) et dans leur contexte actuel (restaurations, état et lieu de conservation). Elles font également l'objet d'une analyse iconographique et stylistique afin de pouvoir rattacher chaque objet à une période. 

sculpture antique dans le cadre de la Bibliothèque de l'enseignement des beaux-arts dirigée par Jules Comte ${ }^{82}$. Ce " petit livre », comme l'avait dit avant lui Collignon pour son propre manuel d'archéologie grecque, se présente comme un ouvrage didactique mais capable «de s'attarder aux débats encore ouverts et aux hypothèses ${ }^{83}$ ». S'inscrivant dans la lignée des manuels déjà écrits par ses ainés - Maxime Collignon, Gaston Maspero ${ }^{84}$, Ernest Babelon $^{85}$ et Jules Martha ${ }^{86}$ - Paris prend appui sur la construction historique qu'ils ont su donner à leurs objets d'étude pour mettre en relief «les traits caractéristiques d'une période, d'une école ou d'un talent ${ }^{87}$ ». Certes, « dans ce commerce familier avec les chefsd'œuvre de l'art antique, dans ces exercices techniques ${ }^{88}$ ", la collection de moulages assure aux enseignements une dimension pratique et une vision concrète et critique de la sculpture. Elle correspond également à la construction scientifique de l'ancien membre de l'École française d'Athènes qui a su compléter sa formation d'épigraphe par un intérêt pour l'archéologie figurée grâce à des fouilles, notamment à Élatée dont la ville et le temple d'Athéna furent le sujet de sa thèse ${ }^{89}$. Nous pouvons observer en dernière analyse, au travers des moulages choisis, que Paris tente de donner une vision élargie de la sculpture archaïque sans la réduire à un simple point de départ d'une histoire dont la sculpture du $V^{e}$ et IV ${ }^{e}$ siècles demeurent les points culminants. En outre, la sélection des moulages accompagne les cours portant sur l'actualité des fouilles archéologiques.

Maxime Collignon, sur la base de ces méthodes d'enseignement, qu'il applique également à la Sorbonne, signe en 1899 un article paru dans la Revue internationale de l'enseignement dans le cadre d'une enquête sur l'enseignement des disciplines ${ }^{90}$. D'une manière générale, la pédagogie appliquée à l'histoire de l'art fait l'objet de réflexions de la part de plusieurs professeurs. Émile Mâle avait déjà publié à ce sujet dans la Revue universitaire ${ }^{91}$. Léon Rosenthal écrira également un pendant à l'article de Collignon afin de souligner la nature « vivante » des objets chez l'historien de l'art en opposition à la vision documentaire de l'historien ${ }^{92}$. Pour chacun, l'objet demeure au centre de la réflexion pédagogique comme il l'est dans la recherche scientifique.

1895, alors que la cour couverte et les trois salles du sous-sol sont désormais occupées par le Musée archéologique, un nouvel aménagement est voté en conseil municipal pour un total de 110000 francs, l'État prenant en charge la moitié des travaux ${ }^{93}$. Désormais, il s'agit d'aménager un hangar devant s'implanter, comme il était prévu pour la cour vitrée, à l'arrière des bâtiments de la faculté des lettres. Si cet espace accueille temporairement les collections de zoologie en attendant qu'elles soient transférées dans le bâtiment construit à cet effet, il devait être dévolu à la présentation des moulages, notamment ceux des frontons d'Olympie « aujourd'hui relégués dans des salles étroites et obscures où il faut pour les admirer, les yeux de la foi ${ }^{94}$ ». Malgré le vote des fonds, la construction n'est pas entreprise pour des raisons qui restent à éclaircir. D'autre part, Pierre Paris, jusque-là accaparé par le Musée archéologique, va consacrer désormais son temps aux fouilles archéologiques en Espagne - la découverte en 1897 de la dame d'Elche constituant son haut fait d'armes.

Bien que le doyen et le conseil des facultés aient soutenu un dernier projet ${ }^{95}$, celui d'un musée commun à la faculté des lettres et à l'école municipale des beaux-arts - idée encore suggérée par Pierre Paris devenu en 1898 directeur de cette école et rêvant d'un « véritable institut artistique ${ }^{96}$ »- l'agencement du Musée archéologique n'évoluera plus. En revanche, ses collections se sont considérablement enrichies, en particulier de photographies - mille dès $1889^{97}$, le fonds s'accroissant les années suivantes. Une 
bibliothèque spécifique à l'histoire de l'art antique a été constituée. L'École française d'Athènes a offert en 1895 à la faculté des lettres de Bordeaux, à l'instar de la Sorbonne et de l'université de Lyon, un ensemble de statuettes de terre cuite provenant des célèbres fouilles d'Edmond Pottier, Salomon Reinach et Alphonse Veyries à Myrina. En 1902, le Ministère de l'Instruction publique a envoyé un lot d'objets provenant des fouilles d'Antinoë ainsi que des fragments de l'art élamite ${ }^{98}$. En outre, le musée du Louvre a déposé au même moment "une série de vases et de fragments de vases antiques permettant de faire, d'après documents originaux, un cours sur la céramique ${ }^{99}$ \%. Enfin, l'orientation des recherches de Pierre Paris a conduit à «faire une bonne place dans les achats nouveaux aux monuments ibériques, grecs, romains, hispano-romains existant dans les Musées espagnols ${ }^{100}$ ». Malgré des espaces peu adaptés - contrairement aux musées qui lui succéderont comme ceux de Montpellier, de Lyon ou de la Sorbonne -, Pierre Paris a su concevoir une collection en concordance avec la construction universitaire de la discipline et les méthodes de diffusion du savoir.

\section{État des lieux des collections archéologiques de l'université de Bordeaux 3}

Les collections de l'ancien Musée archéologique se composent actuellement de 442 moulages, 3312 photographies et d'un ensemble d'objets provenant de fouilles archéologiques ${ }^{101}$. La plupart d'entre elles ont été déposées par l'université de Bordeaux 3 au musée d'Aquitaine qui occupe depuis 1986 le bâtiment de l'ancienne faculté. Si l'on considère que le catalogue des moulages, édité en 1892, contient 280 notices et que l'annuaire des facultés signale, pour la même année, plus de 2000 photographies ${ }^{102}$, on peut penser que le Musée archéologique nous est parvenu, sinon dans son intégralité, du moins dans sa majeure partie. L'état actuel paraît être celui du début du XXe siècle car les moulages n'ont $\mathrm{pu}$, faute de financement et de place, se développer et les épreuves papiers ont été remplacées dès la fin du XIX ${ }^{\mathrm{e}}$ siècle par des verres à projection. Stockés dans les réserves, les objets et les photographies ne sont accessibles ni aux étudiants ni au grand public.

La collection de moulages se compose de 338 œuvres sculptées antiques (ronde-bosse, bas-reliefs, haut-relief, vase) et de 104 décors architecturaux médiévaux (bas-relief, hautrelief ${ }^{103}$. Les œuvres antiques ont fait l'objet de notices rédigées par les élèves de Pierre Paris et éditées en 1892 par Cadoret. Quelques ateliers de moulages ont déjà été identifiés : l'atelier de moulages du musée du Louvre, l'atelier de moulages de l'École des beaux-arts de Paris, l'atelier de moulages de l'École supérieure de Munich. Les moulages de sculptures antiques concernent essentiellement l'art grec (Grèce continentale, Asie Mineure, Grande Grèce) et, dans une moindre mesure, l'art romain. Les moulages des décors médiévaux sont en cours d'identification.

La collection de moulages a fait l'objet d'une double appropriation. D'une part, celle de l'École des beaux-arts qui propose une vision esthétisante fondée sur l'observation des œuvres (études des formes, copie d'antiques, acquisition d'une culture), d'autre part, celle du Musée de sculpture comparée qui diffuse des modèles stéréotypés de décors architecturaux (savoir-faire technique, mode néo-médiévale, répétitions de modèle) ${ }^{104}$. Il en résulte une séparation très nette des savoirs et des formations. L'organisation chronologique et la présentation d'exemples inédits (sculpture ibérique et punique) 
montrent toutefois que Pierre Paris s'éloigne des critères esthétiques qui marquent encore la culture visuelle de l'époque ${ }^{105}$.

39 Les photographies (fig. $\left.\mathbf{n}^{\circ}{ }^{7}\right)\left(\mathbf{f i g} \mathbf{n}^{\circ} \mathbf{8}\right)\left(\right.$ fig. $\left.\mathbf{n}^{\circ} \mathbf{9}\right)$ sont des épreuves sur papier albuminé (2 875), des épreuves sur papier baryté (304) et des photogravures (133) produites à la fin $\mathrm{du} \mathrm{XIX}^{\mathrm{e}}$ et au début du XXe siècle. Les images sont généralement de grands formats (de $18 \times 24 \mathrm{~cm}$ à $40 \times 50 \mathrm{~cm}$ ) et sont collées sur carton. Les sujets concernent des reproductions d'œuvres (1988), des vues d'architecture (1314) et des paysages (10). Les périodes couvrent le Néolithique (11), l'Âge du Bronze (35), l'Antiquité (1 407), le Moyen Âge (538), les époques modernes (1 202) et contemporaines (119). Les lieux concernent l'Égypte, la Palestine, la Syrie, la Turquie, la Grèce, l'Italie, l'Espagne et la France pour les vues d'architecture, les grands musées européens d'Italie, d'Espagne, de France, d'Angleterre, de Belgique, des Pays-Bas, d'Allemagne, d'Autriche et de Russie pour les reproductions d'œuvres d'art.

\section{Figure 7}

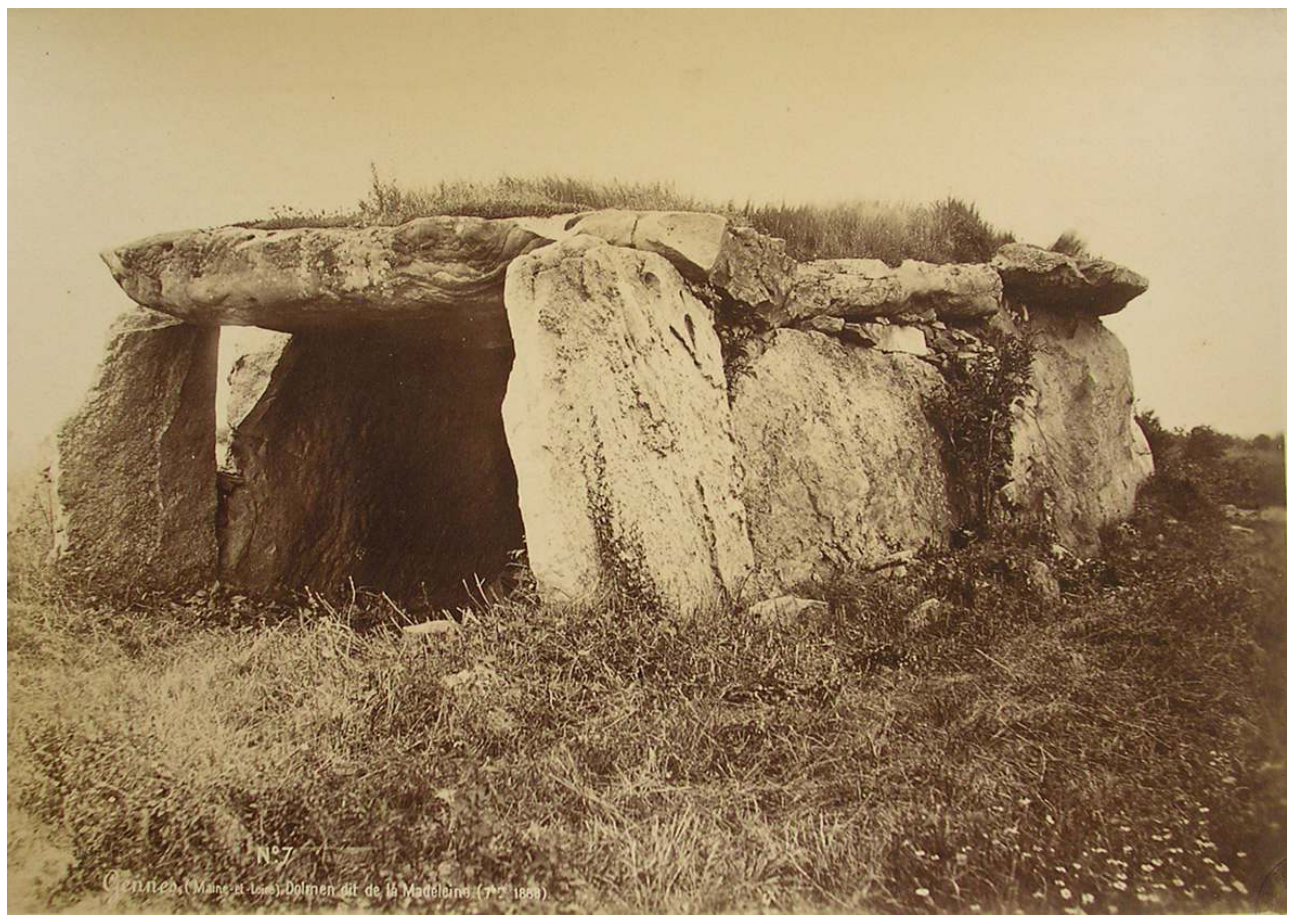

MIEUSEMENT, Séraphin Médéric. Dolmen dit de la Madeleine à Gennes (Maine-et-Loire). 1888, papier albuminé collé sur carton, 22,2 × 27,9 cm.

(c) Université de Bordeaux 3. 
Figure 8

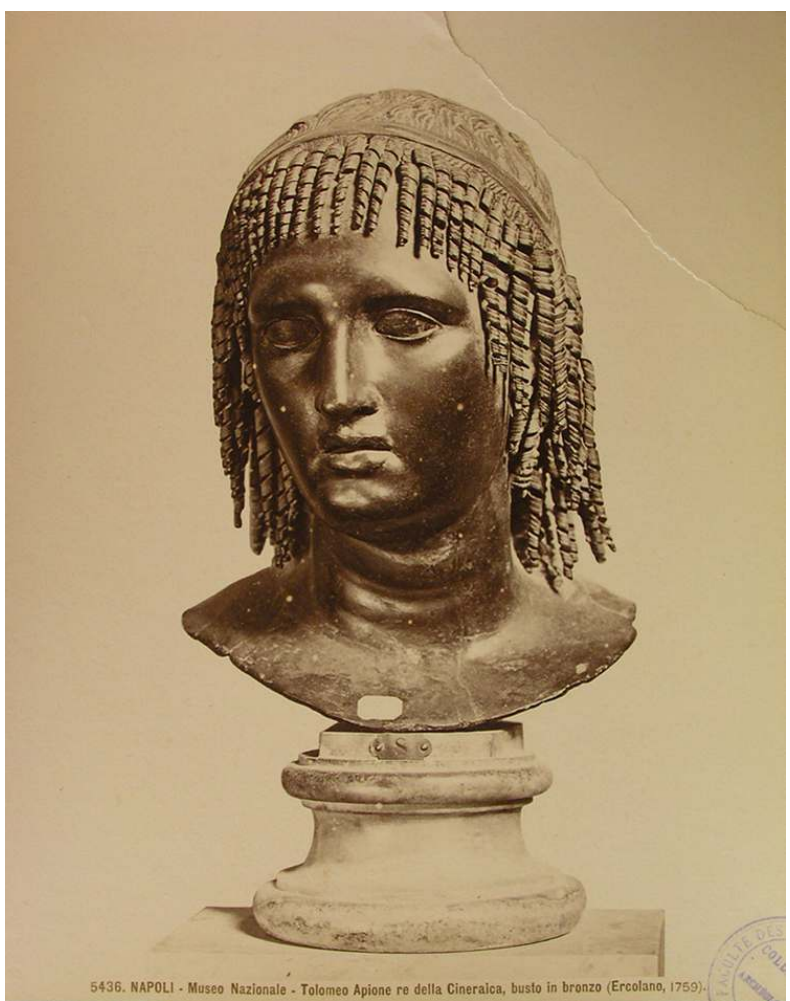

Anonyme. Buste de Ptolémée Apion trouvé à Herculanum en 7759. s.d., papier albuminé collé sur carton, $25,4 \times 20 \mathrm{~cm}$.

(C) Université de Bordeaux 3 


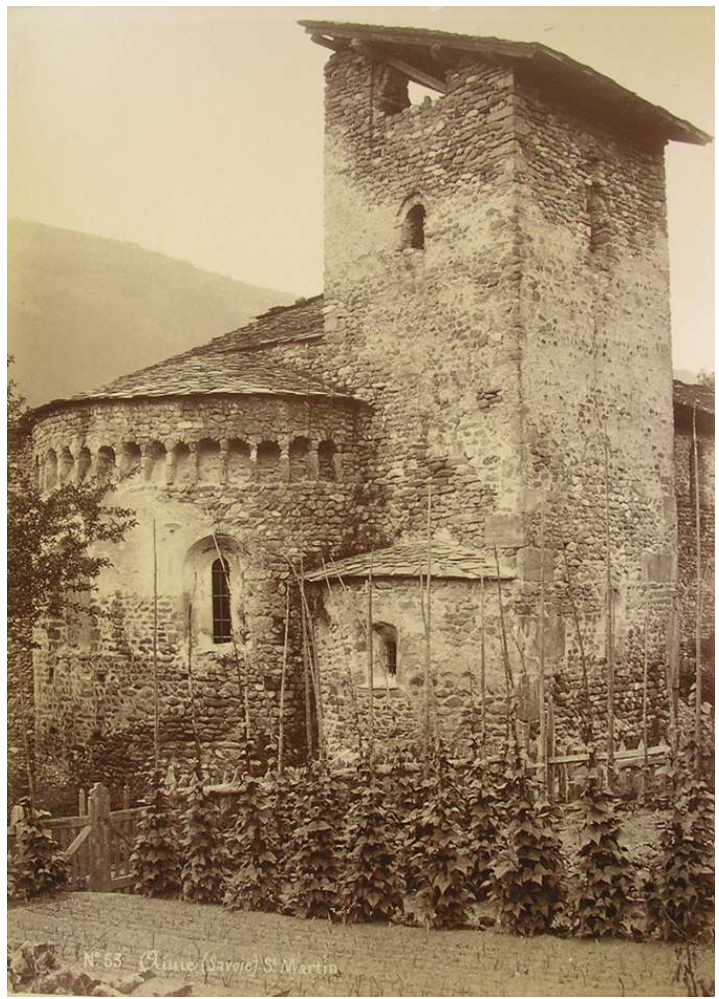

MIEUSEMENT, Séraphin Médéric. Chevet de l'église Saint-Martin à Aime (Savoie). s.d., papier albuminé collé sur carton, $38,3 \times 27 \mathrm{~cm}$.

(c) Université de Bordeaux 3.

Beaucoup de ces images sont anonymes (1 252) mais la plupart sont signées. Certaines ont $\mathrm{pu}$ être attribuées. Les principaux photographes sont Alinari, Brogi et Anderson (architecture, sculpture et peinture de l'Italie antique, médiévale et moderne), Berggren, Sebah et Joaillier (architecture antique, médiévale et moderne en Turquie et en Grèce), Bonfils (architecture antique en Égypte, Palestine, Syrie et Grèce) (fig. $\left.\mathbf{n}^{\circ} \mathbf{1 0}\right)$, Mieusement, Neurdein et Robert (architecture et sculpture médiévale en France) (voir fig. $\mathrm{n}^{\circ} 7, \mathrm{n}^{\circ} 9$ ), Bruckmann (peinture de la pinacothèque de Vienne), Giraudon (peinture et sculpture au Louvre), Hanfstaengl (peinture au Rijksmuseum, au British Museum, au musée de l'Ermitage, à la galerie des beaux-arts de Berlin, à la pinacothèque de Vienne), Laurent (architecture, peinture et sculpture à l'Escorial), Löwy (peinture à la pinacothèque de Vienne), et enfin Ziegler (sculpture au British Museum). À ces vues réalisées par des professionnels, s'ajoutent des images aux techniques plus incertaines faites en amateur. Ce sont des reproductions de sculptures de Rodin mais aussi des vues de l'architecture et de l'artisanat marocain. Ces images ont peut-être été réalisées par Pierre Paris et tirées dans le laboratoire photographique de la faculté. 


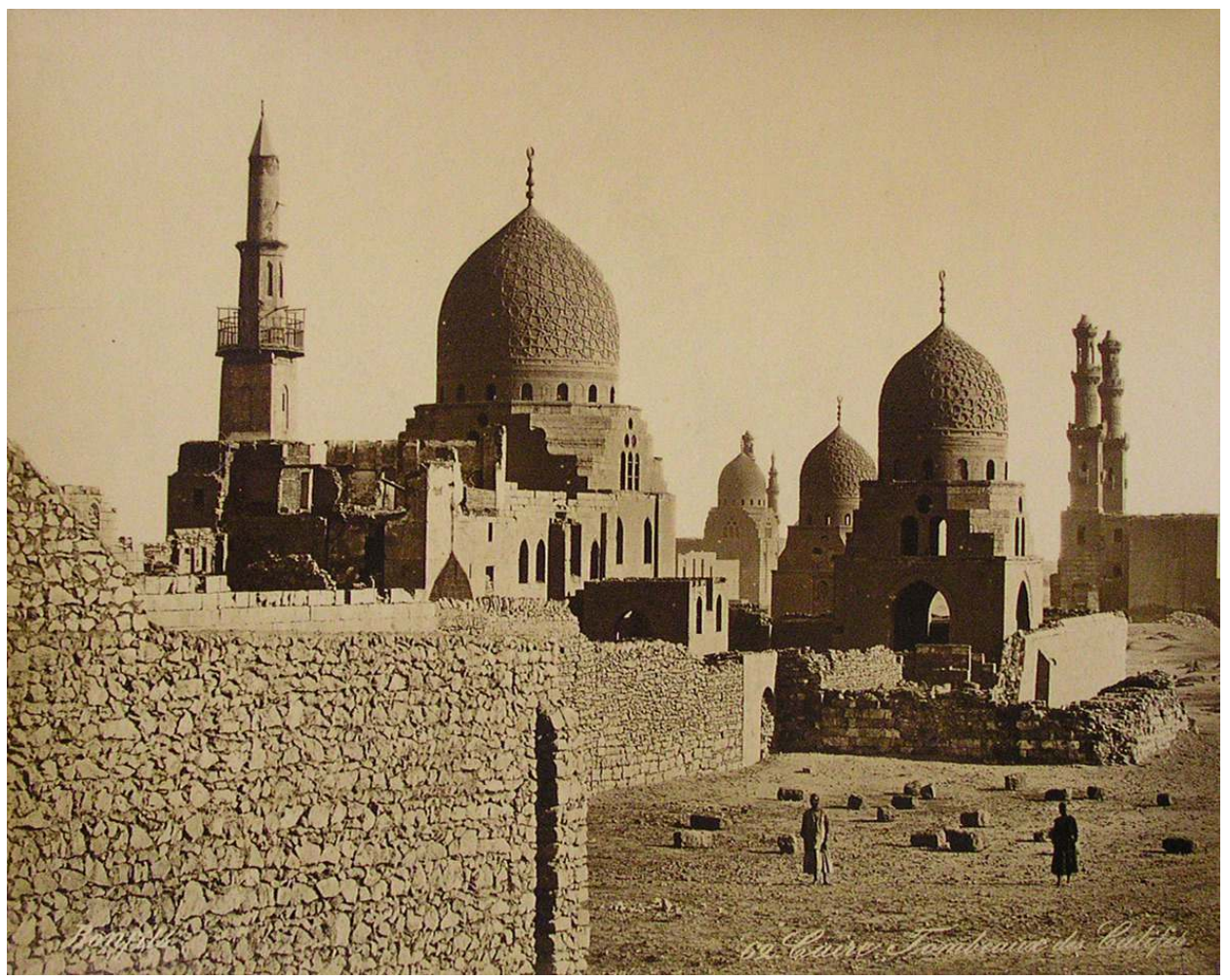

BONFILS, Félix. Tombeaux des Califes au Caire. s.d., papier albuminé collé sur carton, 22,2 × 27,9 cm.

(c) Université de Bordeaux 3 .

La collection de photographies s'inspire des fonds iconographiques des grandes institutions nationales tant sur le plan du savoir (Institut de France, Commission des monuments historiques, société de géographie) que de l'enseignement (École des beauxarts, Musée de sculpture comparée, École des Ponts et Chaussées). Elle est issue de la production des grandes maisons d'édition photographique qui se sont constituées vers 1860. Ces maisons s'adressent avant tout à une clientèle de voyageurs, de curieux et de collectionneurs. Leur imagerie, particulièrement abondante, donne accès à une multitude d'œuvres reproduites selon des points de vue variés. Diffusées par catalogue, ces banques d'images sont accessibles aux professeurs d'université qui constituent une nouvelle clientèle. Cependant, l'esthétique de ces images qui doit satisfaire plusieurs publics est souvent ambiguë. Elle oscille entre l'évocation pittoresque (perspectives fuyantes, effets lumineux) et le document scientifique (frontalité, lisibilité).

Si les moulages semblent renvoyer à une conception esthétisante de l'histoire de l'art et à un savoir-faire pratique, leur association avec la photographie rend compte d'une toute autre approche. Ces deux médiums relèvent bien plus des techniques d'empreintes que de la reproduction formelle. En ce sens, ils témoignent d'un renouvellement méthodologique basé sur une approche scientifique (rapport au réel, volonté d'objectivité, analyse de séries) ${ }^{106}$. L'enseignement reprend ainsi le modèle scientifique évoqué précédemment: l'érudition (le cours, la bibliothèque), l'observation (les moulages, les photographies), l'expérimentation (les travaux pratiques, le catalogue).

43 Au-delà d'une simple illustration des cours de Pierre Paris, ce premier musée archéologique et universitaire de France est un des éléments constitutifs d'une histoire de 
l'art en construction. Révélateur des politiques municipales concernant l'enseignement supérieur, il apporte des informations déterminantes sur les personnes qui sont à l'origine de notre système universitaire. Les collections archéologiques témoignent de l'élargissement progressif des frontières de la discipline (époques, lieux, cultures) et d'un profond renouvellement du regard. Les moulages et les photographies permettent de dissocier, de comparer ou de mettre en série les différents objets d'études. La photographie par l'étendue de ses thèmes, l'abondance des images et la variété des points de vue, apparaît comme un véritable outil de pensée et fait l'objet, au tournant du siècle, de nombreuses analyses d'historiens de l'art ${ }^{107}$.

\section{Le statut du Musée archéologique hier et aujourd'hui}

Lors de la construction du bâtiment, et peu de temps après son achèvement, le photographe Alphonse Terpereau (1839-1897) a réalisé plusieurs campagnes photographiques de la faculté des lettres et des sciences ${ }^{108}$. Particulièrement démonstratifs, les différents tirages répondent à des lectures différentes selon les commanditaires. Les archives de la ville conservent des photographies du chantier de construction qui témoignent de la puissance du conseil municipal et de la valeur de ses services. La faculté des lettres a, quant à elle, conservé jusqu'à aujourd'hui un album photographique prestigieux où les vues des façades monumentales du bâtiment cohabitent avec les vues intérieures. Le musée des moulages y est représenté selon trois angles différents (voir fig. $n^{\circ} 4, n^{\circ} 5$ et $n^{\circ} 6$ ) aux côtés des laboratoires de physique et de chimi ${ }^{109}$. D'autres clichés réalisés par le même photographe avaient d'ailleurs été encadrés ${ }^{110}$. Certains de ces tirages ont également fait l'objet, à la demande du ministère de l'Instruction publique, d'un accrochage lors des Expositions universelles de Paris en $1889^{111}$ et de Chicago en $1893^{112}$. Ils y figuraient aux côtés des écrits des professeurs afin de conférer à l'université française le prestige recherché.

Les années 1960 - de manière visible avec les graffitis de mai 1968 qui sont venus maculer les plâtres - marquent un désintérêt pédagogique pour cette collection qui demeurera utilisée néanmoins par certains enseignants ${ }^{113}$. Le programme des bâtiments de l'université de Bordeaux 3 n'intégrant pas d'espace spécifique pour cette collection archéologique, son usage se perd définitivement. Les épreuves sur papier ont été également délaissées en raison de l'accroissement du nombre d'étudiants au début du XX siècle qui a conduit à utiliser les verres à projection. Lors du transfert sur le campus en 1969, les statuettes de Myrina acquièrent en revanche un statut privé, mais honorifique, en étant exposées dans le bureau de Jean Marcadé, professeur d'archéologie et d'histoire de l'art antique, dans un meuble conçu à cet effet. Ces collections font aujourd'hui l'objet d'une véritable redécouverte grâce à un programme de recherches de la Région Aquitaine portant sur le patrimoine artistique universitaire de l'université de Bordeaux (Centre de recherches F.-G. Pariset, E.A. 538). Les données, une fois inventoriées et numérisées, seront notamment accessibles par le biais de la bibliothèque numérique du Service commun de documentation de Bordeaux 3 et sous la forme d'expositions virtuelles. Dans un contexte de réformes universitaires, ces objets constituent une véritable richesse qu'il s'agit de faire partager et de sauvegarder. 
NOTES

1. Les intitulés des fonds d'archives ont été nommés en toutes lettres lors de la première occurrence, puis sous la forme d'un acronyme pour les suivantes.

- COUAT, Auguste. «Le Musée d'Alexandrie sous les premiers Ptolémées ». Annales de la faculté des lettres de Bordeaux, 1879, t. I, p. 7-28, 17-18.

2. - Ce sont les collections de Lyon, Montpellier, Strasbourg et Paris qui sont les mieux citées en raison des études menées et, parfois, de leur visibilité dans un cadre muséal. Voir notamment MOSSIÈRE, Jean-Claude. "Les musées des moulages ». La Lettre de l'OCIM, 1996, n44, p. 10-13 ; MARTINEZ, Jean-Luc. « La collection de moulages : un musée pédagogique ». Dans TEXIER, Simon (dir.). L'Institut d'art et d'archéologie. Paris. 1932. Paris : A. et J. Picard, 2005, p. 93-104 ; MORINIÈRE, Soline. Le Musée des Moulages de la Faculté des Lettres de Montpellier. Origine et développement d'une collection universitaire (1890-1904). Master 1 Histoire de l'art. Paris : École du Louvre, 2010.

3. - ORGOGOZO, Chantal (dir.). Le musée d'Aquitaine, Bordeaux. Paris : Musées et monuments de France, 1992 ; HUBERT, François et ZIEGLÉ, Anne. L'histoire du Musée d'Aquitaine. Anabases. Traditions et Réceptions de l'Antiquité, octobre 2010, n¹2, p. 230-238.

4. - CLAVEL, Elsa. La faculté des lettres de Bordeaux de 1886 à 1940. T.E.R. d'histoire contemporaine. Bordeaux : Université Michel de Montaigne, 1993 ; POUX, Ludovic. Les palais universitaires. T.E.R. d'histoire contemporaine. Bordeaux: Université Michel de Montaigne, 1993; CADILHON, François, LACHAISE, Bernard. et LEBIGRE, Jean-Michel. Histoire d'une université bordelaise: Michel de Montaigne, faculté des arts, faculté des lettres, 1441-1999. Bordeaux: Presses universitaires de Bordeaux, 1999.

5. - THERRIEN, Lyne. L'histoire de l'art en France. Genèse d'une discipline universitaire. Paris : éditions du C.T.H.S., 1998 ; RECHT, Roland. «Le moulage et la naissance de l'histoire de l'art ». Dans Le musée de sculpture comparée: Naissance de l'histoire de l'art. Paris : Monum-Centre des monuments nationaux, 1999, p. 46-53.

6. - Voir la note 2. Voir aussi : BARBANERA, Marcello. "Les collections de moulages au XIX siècle : étapes d'un parcours entre idéalisme, positivisme et esthétisme ». Dans LAVAGNE, Henri et QUEYREL, François (dir.). Les moulages de sculptures antiques et l'histoire de l'archéologie. Actes du colloque international, 24 octobre 1997. Genève: Droz - Paris: Champion, 2000, p. 57-73; SWENSON, Astrid. «Musées de moulages et politiques patrimoniales. Regards croisés sur la France, l'Allemagne et l'Angleterre au XIX ${ }^{\mathrm{e}}$ siècle ». Dans ROLLAND, Anne-Solène et MURAUSKAYA, Hanna (dir.). Les Musées de la Nation. Créations, transpositions, renouveaux. Europe, XIX ${ }^{e}$-XXI ${ }^{e}$ siècles. Actes de colloque, 5, 6 et 7 décembre 2007. Paris : L'Harmattan, 2008, p. 205-219.

7. - BÉGUERIE, Catherine. La Naissance de l'enseignement de l'histoire de l'art à Bordeaux. Master 2 Histoire de l'art. Bordeaux: Université Michel de Montaigne, 2003; BÉGUERIE, Catherine. «L'Enseignement de l'histoire de l'art à Bordeaux. Premiers cours, premiers professeurs: l'émergence d'une discipline ». Revue archéologique de Bordeaux, année 2004, t. XCV, p. 225-238.

8. - MORINIÈRE, Soline. La collection de moulages d'antiques et médiévaux de la Faculté des Lettres de Bordeaux en dépôt au musée d'Aquitaine : étude documentaire, conservation et perspectives. Master 2 professionnel. Paris : École du Louvre, 2011. Florent Miane, post-doctorant sur le programme "Région", a réalisé le récolement de l'ensemble du fonds photographique en dépôt au musée d'Aquitaine. Des verres à projection conservés à l'université sont en cours d'inventaire.

9. - L'université de Bordeaux ayant été supprimée pendant la Révolution, en tant que corporation définie par des privilèges, seule la faculté des lettres avait été rétablie pendant le Premier Empire 
(décret du 17 mars 1808), puis de nouveau supprimée sous la Restauration (ordonnance du 17 février 1815).

10. - BEAUCHAMP, Arthur Marais de. Recueil des lois et règlements sur l'enseignement supérieur: comprenant les décisions de la jurisprudence et les avis des conseils de l'Instruction publique et du conseil d'État. Paris : Delalain frères, 1880-1915, p. 783.

11. - Archives Municipales de Bordeaux (AMB), délibérations du conseil municipal, $12 \mathrm{D} 20,30$ octobre 1838. Les premiers aménagements concernent l'achat de mobilier pour le cabinet de physique et pour les collections de minéralogie, un réservoir et des tuyaux dans la salle de chimie, un fourneau dans l'amphithéâtre des sciences, un meuble pour la bibliothèque, des tentures dans les amphithéâtres des sciences et des lettres pour atténuer leur trop grande sonorité.

12. - AMB, délibérations du conseil municipal, 12 D 19, 22 janvier 1838.

13. - AMB, délibérations du conseil municipal, 12 D 20, 30 octobre 1838.

14. - AMB, délibérations du conseil municipal, 12 D 21, 15 février 1840. La collection Roger, décrite comme l'une des plus belles de France, se compose de 15000 coléoptères et de 7300 lépidoptères.

15. - RAYET, Georges. "Histoire de la faculté de Bordeaux". Actes de l'Académie des sciences et belles-lettres, 1897, t. 340, p.97. Ce sont des roches et fossiles des environs de Paris (don du Muséum d'histoire naturelle, 95 numéros) ; des roches d'Auvergne (200 échantillons) ; une série de roches de l'Etna (rapportée par Élie de Beaumont en 1834 et donnée par Giacinto Provana, comte de Collegno); une collection de fossiles et de roches caractéristiques classés par la méthode Bronn (900 numéros) ; une collection de fossiles tertiaires du Piémont et de minéraux des Alpes, du Piémont et de la Savoie donnée par De Collegno ; une série de roches des Pyrénées. Ces collections sont rapidement augmentées par des dons (les échantillons du professeur Rolin, les échantillons de Delbos, les moulages du Muséum de Paris), par des achats divers (minéraux et fossiles) et par l'acquisition de la collection Banon (fossiles de faluns). Voir : CAHUZAC, Bruno. «Le Docteur Grateloup, naturaliste précurseur en paléontologie landaise. I -Aperçu sur sa vie et grands traits de son oeuvre scientifique ». Bulletin de la Société de Borda, 2001, 126 année, 462 (3), p. 367-414. ARMAND, Dominique, DE LA NOË, Jérôme, BESSOU, Maryelle et al. « Les collections scientifiques de l'université Bordeaux 1. Sciences et Technologies ». Actes du 134 ${ }^{e}$ Congrès national des Sociétés historiques et scientifiques, avril 2009. Paris : C.T.H.S., à paraître.

16. - RAYET, Georges. "Histoire de la faculté de Bordeaux ». Actes de l'Académie des sciences et belles-lettres, 1897, t.340, p. 90-91. Ce sont une machine électrique à frottement, une machine électromagnétique, une machine pneumatique, une machine d'Atwood, un banc de Melloni, une série de lentilles et de miroirs, un appareil d'acoustique, un théodolite de Gambey, une boussole d'inclinaison, une boussole de déclinaison, un banc de diffraction, une série d'appareils d'induction et des balances (20 000 francs).

17. - AMB, correspondances et délibérations du conseil municipal, $1057 \mathrm{R} 1,27$ juillet 1840 : achat d'une collection zoologique (48 oiseaux et une tortue, 694 francs) ; 1057 R 1, 2 octobre 1840 : achat de 28 instruments de chirurgie pour la zoologie (59,65 francs) ; $1057 \mathrm{R} \mathrm{1,} 6$ octobre 1840 : achat d'une collection zoologique (43 animaux et 46 éléments de présentation, 228,60 francs); 1057 R 1, 21 janvier 1841 : achat de 284 articles de laboratoire pour la zoologie (150,61 francs); 1057 R 1, 15 juin 1841 : achat de onze produits chimiques pour le laboratoire d'anatomie $(36,30$ francs); 1057 R 1, 15 mais 1842: achat d'armoires vitrées pour la zoologie (250,77 francs); $12 \mathrm{D} 23,5$ décembre 1842 : achat de divers objets indispensables à l'enseignement de la zoologie (épingle à insecte, boîte à insecte, filet à papillon... 1518 francs) et des animaux naturalisés locaux, étrangers, rares (oiseaux de Nouvelle-Hollande, tamanoir... 926 francs).

18. - AMB, correspondances et délibérations du conseil municipal, $1057 \mathrm{R} 1,4$ octobre 1841 : achat de cinq instruments d'astronomie (cercle mural, lunette méridienne, lunette équatoriale, modèles d'anneaux de Saturne, cercle à réflexion, 2075 francs); 1057 R 1, 2 mai 1842: achat 
d'une boussole de déclinaison (1 800 francs) ; $1057 \mathrm{R} \mathrm{1,} 28$ décembre 1842 : achat d'une lunette astronomique avec un objectif de $16 \mathrm{~cm}$ (2 600 francs).

19. - AMB, délibérations du conseil municipal, 12 D 22, 4 février 1842.

20. - Jean-Joseph-Benoît Abria est né en 1811. Il est nommé professeur de physique à la faculté des sciences de Bordeaux en 1839 et devient doyen de la faculté des sciences en 1845.

21. - AMB, correspondances du conseil municipal, $6889 \mathrm{M} \mathrm{7,} 7$ juillet 1858.

22. - AMB, correspondances du conseil municipal, 6889 M 7, 15 décembre 1862.

23. - AMB, délibérations du conseil municipal, 12 D 19, 22 janvier 1838.

24. - RAYET, Georges. «Histoire de la faculté de Bordeaux ». Actes de l'Académie des sciences et belles-lettres, 1897, t. 340, p. 33.

25. - COUSIN, Victor. Rapport sur l'état de l'instruction publique dans quelques pays d'Allemagne et particulièrement en Prusse. Paris-Strasbourg: Levrault, 1833; RENAN, Ernest. Questions contemporaines. Paris : Michel-Lévy frères, 1867.

26. - AMB, correspondances du conseil municipal, 6889 M 8, 17 octobre 1875.

27. - AZAM, Eugène. De la décentralisation universitaire et pourquoi Bordeaux doit avoir son université. Bordeaux-Paris : Féret-Masson, 1871.

28. - AMB, correspondances du conseil municipal, 6889 M 8, 9 février 1875.

29. - AMB, correspondances du conseil municipal, $6889 \mathrm{M} \mathrm{8,14}$ mars 1875 . Il reprend les projets des années 1840 en rajoutant une salle pour l'électricité et une salle pour la photographie.

30. - AMB, correspondances du conseil municipal, $6889 \mathrm{M} \mathrm{8,} \mathrm{octobre} 1875$.

31. - Né en 1823, professeur d'astronomie à la faculté des sciences de Bordeaux en 1858, Gaston Lespiault devient membre du conseil municipal en 1874, puis membre du Conseil supérieur de l'instruction publique en 1880, et enfin doyen de la faculté des sciences de Bordeaux en 1886.

32. - AMB, correspondances du conseil municipal, $6889 \mathrm{M} \mathrm{1,} 17$ octobre 1876. L'emprunt doit couvrir : la construction de la faculté de médecine, le regroupement des facultés de théologie, sciences et lettres, la construction d'un observatoire astronomique, la réorganisation du service des eaux, l'achat de maisons, le remboursement d'emprunts précédents, le déplacement du lycée et la construction de l'église Saint-Louis. Voir aussi AMB, correspondances du conseil municipal, $1050 \mathrm{R} \mathrm{1,} 17$ octobre 1876 (rapport à la commission des finances). L'emprunt et le devis seront revus à la baisse, l'État autorisera la ville à emprunter 5500000 francs (AMB, correspondances du conseil municipal, $6889 \mathrm{M} \mathrm{8,13}$ juillet 1877) le devis des facultés sera fixé à 1400000 francs (AMB,

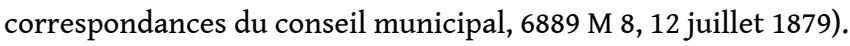

33. - AMB, correspondances du conseil municipal, 6889 M 8, 9 février 1875. L'enseignement des sciences développe des applications industrielles et crée des laboratoires privés; celui des lettres forme les professeurs des collèges et améliore la rhétorique des avocats ; d'une manière générale, la présence de grands scientifiques améliore la réputation de la ville.

34. - Le calcul est simple : 850 étudiants dépensent 2550000 francs par an ; 38 fonctionnaires (six en théologie, douze en droit, six en sciences, cinq en lettres, huit en médecine, un recteur) dépensent 304000 francs par an; 41 employés (commis, préparateurs, appariteurs, aides des chefs de travaux) dépensent 82000 francs par an. Au total, ils rapportent à la ville: 2936000 francs par an.

35. - Né en 1808, professeur de littérature à la faculté des lettres de Bordeaux en 1838, PhilippeJacques Roux devient doyen de la faculté des lettres en 1875.

36. - AMB, correspondances du conseil municipal, 6889 M 8, 31 mars 1879.

37. - RENAUT, Alain. "Louis Liard et l'élitisme républicain». Romantisme, 1995, vol. 2, n88, p. 85-100. Né en 1846, professeur de philosophie à la faculté des lettres de Bordeaux en 1874, Louis Liard est nommé recteur de l'université de Caen en 1880, puis directeur de l'enseignement supérieur de 1884 à 1902. 
38. - JOCKEY, Philippe. "Maxime Collignon ». Dans SÉNÉCHAL, Philippe et BARBILLON, Claire (dir.). Dictionnaire critique des historiens de l'art actifs en France de la Révolution à la Première guerre mondiale. Paris : INHA, 2009. http://www.inha.fr/spip.php?article3227 [consulté le 10/05/11].

39. - ROUILLARD, Pierre. «Pierre Paris ». Dans SÉNÉCHAL, Philippe et BARBILLON, Claire (dir.). Dictionnaire critique des historiens de l'art actifs en France de la Révolution à la Première guerre mondiale. Paris : INHA, 2009. http://www.inha.fr/spip.php?article2480 [consulté le 10/05/11].

40. - RENAUT, Alain. « Louis Liard et l'élitisme républicain ». Romantisme, 1995, vol. 2, n88.

41. - Louis Liard fut membre du conseil municipal de la ville de Bordeaux et adjoint au maire délégué à l'instruction publique de 1878 à 1881.

42. - AMB, correspondances du conseil municipal, 6889 M 8, 12 juillet 1879. Rapport présenté au Conseil Municipal sur les plans et devis des nouvelles Facultés de Théologie, des Sciences et des Lettres, par M. L. Liard, Annexe - programme des constructions, p. 6.

43. - Ibid. «Une salle pour les collections d'archéologie (Cette salle pourrait être rendue inutile par une disposition qui permettrait de placer les moulages d'archéologie soit dans un pas perdu, soit dans un vestibule) ».

44. - AMB, correspondances du conseil municipal, 6889 M 8, 5 février 1886. Lettre de Charles Durand à Auguste Couat.

45. - Auguste-Henri Couat, dit Auguste Couat, né en 1846, normalien, professeur de rhétorique dans plusieurs lycées de province, fut nommé en 1878 titulaire de la chaire de littérature grecque à la faculté des lettres de Bordeaux. Il en devint le doyen le 12 mai 1881. De 1884 à 1886, il fut membre du conseil municipal de Bordeaux et adjoint au maire chargé de l'instruction publique. Il sera élu membre du Conseil supérieur de l'instruction publique par les facultés des lettres en avril 1884 .

46. - AMB, correspondances du conseil municipal, 6889 M 8, 8 janvier 1886. Le conseiller municipal Abel Jay au maire de la ville de Bordeaux.

47. - OUVRÉ, Henri-François. «Discours de M. le Recteur». Rentrée solennelle des Facultés des Sciences et des Lettres de Bordeaux (25 novembre 1886) et comptes-rendus des travaux des Facultés, Année scolaire 1886-1887. Bordeaux : Vve Cadoret, 1886, p. 9-24, 19.

48. - MORINIÈRE, Soline. La collection de moulages d'antiques et médiévaux de la Faculté des Lettres de Bordeaux en dépôt au musée d'Aquitaine: étude documentaire, conservation et perspectives. Master 2 professionnel. Paris : École du Louvre, 2011.

49. - La première chaire d'archéologie avait été créée quelques mois plus tôt à Paris (Sorbonne) par un décret du 6 mars 1876. Elle est occupée par Georges Perrot jusqu'en 1900, Maxime Collignon lui succédant alors.

50. - ROUX, Philippe-Jacques. « Rapport du doyen de la faculté de lettres ». Rentrée solennelle des Facultés de Théologie, des Sciences et des Lettres et de l'école préparatoire de médecine et de pharmacie, 15 novembre 1876. Bordeaux : Vve Cadoret, 1876, p. 45-67, 46.

51. - COLLIGNON, Maxime. Faculté des lettres de Bordeaux. Cours d'antiquités grecques et latines. De l'archéologie grecque. Leçon d'ouverture du 15 janvier 1877. Bordeaux : G. Gounouilhou, 1877, p. 3-23, 3.

52. - Rentrée solennelle des Facultés de Théologie, des Sciences et des Lettres et de l'école préparatoire de médecine et de pharmacie, 25 novembre 1878. Bordeaux : V ${ }^{\text {ve }}$ Cadoret, 1878, p. 81-82.

53. - COLLIGNON, Maxime. "L'enseignement de l'archéologie classique et les Collections de moulages dans les Universités allemandes ». Revue internationale de l'enseignement, janvier-juin 1882 , t. 3, p. 256-270.

54. - COLLIGNON, Maxime. "L'enseignement de l'archéologie classique et les Collections de moulages dans les Universités allemandes ». Revue internationale de l'enseignement, janvier-juin 1882 , t. 3., p. 266-267. 
55. - COLLIGNON, Maxime. "L'archéologie à l'Université de Paris». Revue internationale de l'enseignement, janvier-juin 1899, t. 37, p. 193-198, 197.

56. - THERRIEN, Lyne. L'histoire de l'art en France. Genèse d'une discipline universitaire. Paris : éditions du C.T.H.S., 1998, p. 243-251 ; JOCKEY, Philippe. « Maxime Collignon ». Dans SÉNÉCHAL, Philippe et BARBILLON, Claire (dir.). Dictionnaire critique des historiens de l'art actifs en France de la Révolution à la Première guerre mondiale. Paris : INHA, 2009.

57. - Collignon fait figure de précurseur en publiant en 1881 un Manuel d'archéologie grecque dans la collection de la «Bibliographie de l'enseignement des beaux-arts » chez l'éditeur Quantin.

58. - COLLIGNON, Maxime. "L'archéologie à l'Université de Paris». Revue internationale de l'enseignement, janvier-juin 1899, t. 37, p. 196.

59. - THERRIEN, Lyne. L'histoire de l'art en France. Genèse d'une discipline universitaire. Paris : éditions du C.T.H.S., 1998, p. 243-249.

60. - THERRIEN, Lyne. L'histoire de l'art en France. Genèse d'une discipline universitaire. Paris : éditions du C.T.H.S., 1998, p. 262.

61. - THERRIEN, Lyne. L'histoire de l'art en France. Genèse d'une discipline universitaire. Paris : éditions du C.T.H.S., 1998, p. 350-351 ; JOCKEY, Philippe. «Camille Jullian ». Dans SÉNÉCHAL, Philippe et BARBILLON, Claire (dir.). Dictionnaire critique des historiens de l'art actifs en France de la Révolution à la Première guerre mondiale, Paris : INHA, 2009, http://www.inha.fr/spip.php?article2377 [consulté le 10/05/11].

62. - Archives départementales de la Gironde (ADG), V111 liasse 213, enseignants faculté (dossier Pierre Paris).

63. - COUAT, Auguste. «Rapport du doyen de la faculté des lettres ». Rentrée solennelle des Facultés des Sciences et des Lettres de Bordeaux (25 novembre 1886) et comptes-rendus des travaux des Facultés, Année scolaire 1886-1887. Bordeaux : Vve Cadoret, 1886, p. 85-102, 88-89.

64. - ESPINAS, Alfred. « Rapport du doyen de la faculté des lettres ». Comptes-rendus des travaux des Facultés, Année scolaire 1886-1887. Bordeaux : Vve Cadoret, 1887, p. 86-103, 92.

65. - ESPINAS, Alfred. « Rapport du doyen de la faculté des lettres ». Comptes-rendus des travaux des Facultés, Année scolaire 1886-1887. Bordeaux : Vve Cadoret, 1887, p. 93.

66. - ADG, V111 liasse 213. Lettre de Louis Liard au Recteur, Paris, 6 août 1887 ; lettre de Louis Liard au Recteur, Paris, 7 octobre 1887.

67. - Archives du Rectorat de Bordeaux (ARB), conseil général des facultés de Bordeaux, R 23, séance du mardi 8 mars 1887 , f 36 .

68. - ARB, conseil général des facultés de Bordeaux, $R$ 23, séance du mardi 15 novembre 1887, f 56-57.

69. - ESPINAS, Alfred. «Rapport annuel au Conseil académique et Conseil général des Facultés ». Comptes-rendus des travaux des Facultés de droit, de médecine, des sciences et des lettres, Année scolaire 1887-1888. Bordeaux : Vve Cadoret, 1888, p. 83-100, 92.

70. - ARB, conseil général des facultés de Bordeaux, $\mathrm{R} 23$, séance du mardi 15 décembre 1891, f 156.

71. - ARB, conseil général des facultés de Bordeaux, $\mathrm{R} 23$, séance du mardi 15 décembre 1891 , f 156.

72. - AMB, correspondances du conseil municipal, $6889 \mathrm{M} \mathrm{8,} 18$ janvier 1893. Lettre du doyen Stapfer au Recteur.

73. - AMB, correspondances du conseil municipal, 6889 M 8, 14 novembre 1893. Lettre de Pierre Paris au doyen Staper.

74. - AMB, délibérations du conseil municipal, BIB M 2 25, 11 décembre 1894.

75. - Archives de l'Observatoire de Bordeaux, Lettre de Pierre Paris au Doyen, 22 février 1895.

76. - UNIVERSITÉ DE BORDEAUX. Livret-guide, 1906-1907. Bordeaux : Vve Cadoret, 1906, p. 92.

77. - BÉGUERIE, Catherine. La Naissance de l'enseignement de l'histoire de l'art à Bordeaux. Master 2 Histoire de l'art. Bordeaux: Université Michel de Montaigne, 2003, vol. I, p. 31-33 ; BÉGUERIE, 
Catherine. «L'Enseignement de l'histoire de l'art à Bordeaux. Premiers cours, premiers professeurs : l'émergence d'une discipline ». Revue archéologique de Bordeaux, année 2004, t. XCV, p. 228.

78. - UNIVERSITÉ DE FRANCE. Annuaire des facultés de Bordeaux, 1889-1890. Bordeaux : Vve Cadoret, 1889, p. 123.

79. - Alfred Espinas est né en 1844. Normalien, il devient en 1881 professeur titulaire de la faculté des lettres de Bordeaux. Il en est le doyen à partir de 1887.

80. - PARIS, Pierre (dir.). Musée archéologique, Catalogue méthodique des moulages des cuvres de sculpture grecque, rédigé par un groupe d'étudiants sous la direction de Pierre Paris. Bordeaux : Vve Cadoret, 1892 (préface non paginée).

81. - PARIS, Pierre et LA VILLE DE MIRMONT, Henri de. «Le musée de moulages de Bordeaux ». Revue internationale de l'enseignement, juillet à décembre 1899, t. 38, p. 513-515.

82. - MARTIN, Annabelle. "Jules Comte ». Dans SÉNÉCHAL, Philippe et BARBILLON, Claire (dir.). Dictionnaire critique des historiens de l'art actifs en France de la Révolution à la Première guerre mondiale. Paris : INHA, 2009. http://www.inha.fr/spip.php?article2251 [consulté le 10/05/11].

83. - PARIS, Pierre. La Sculpture antique. Paris : Maison Quantin, 1889, p. 6.

84. - DAVID, Élisabeth. « Gaston Maspero ». Dans SÉNÉCHAL, Philippe et BARBILLON, Claire (dir.). Dictionnaire critique des historiens de l'art actifs en France de la Révolution à la Première guerre mondiale. Paris : INHA, 2009. http://www.inha.fr/spip.php?article2446 [consulté le 10/05/11].

85. - BODENSTEIN, Felicity. "Ernest Babelon ». Dans SÉNÉCHAL, Philippe et BARBILLON, Claire (dir.). Dictionnaire critique des historiens de l'art actifs en France de la Révolution à la Première guerre mondiale. Paris : INHA, 2009. http://www.inha.fr/spip.php?article2177 [consulté le 10/05/11].

86. - GAULTIER, Françoise, MATHIEUX, Néguine. "Jules Martha ». Dans SÉNÉCHAL, Philippe et BARBILLON, Claire (dir.). Dictionnaire critique des historiens de l'art actifs en France de la Révolution à la Première guerre mondiale. Paris : INHA, 2009. http://www.inha.fr/spip.php?article2443 [consulté le 10/05/11].

87. - PARIS, Pierre. La Sculpture antique. Paris : Maison Quantin, 1889, p. 5.

88. - ESPINAS, Alfred. « Rapport annuel au Conseil académique et Conseil général des Facultés ». Comptes-rendus des travaux des Facultés de droit, de médecine, des sciences et des lettres, Année scolaire 1886-1887. Bordeaux : Vve Cadoret, 1887, p. 86-103, 93.

89. - MARCADÉ, Jean. «La vie et l'œuvre de Pierre Paris ». Célébration du centenaire de la naissance de Pierre Paris et de Georges Radet, 10 et 11 mars 1961. [Bordeaux] : s.é., 1963, p. 12-30, 17-19.

90. - COLLIGNON, Maxime. "L'archéologie à l'Université de Paris». Revue internationale de l'enseignement, janvier à juin 1899, t. 37, p. 193-198.

91. - MÂLE, Émile. «L'enseignement de l'histoire de l'art dans l'Université ». Revue universitaire, 1894, troisième année, tome premier, p. 10-20.

92. - ROSENTHAL, Pierre. «L'enseignement de l'histoire de l'art». Revue internationale de l'enseignement, janvier à juin 1899, t. 37, p. 299-306.

93. - AMB, compte-rendu, BIB M 3 26, 9 juillet 1895.

94. - AMB, compte-rendu, BIB M 3 26, 9 juillet 1895.

95. - PARIS, Pierre et LA VILLE DE MIRMONT, Henri de. «Le musée de moulages de Bordeaux ». Revue internationale de l'enseignement, juillet à décembre 1899, t. 38, p. 514-515; ARB, conseil général des facultés, R 24, 13 mars 1909, f०262.

96. - PARIS, Pierre et LA VILLE DE MIRMONT, Henri de. «Le musée de moulages de Bordeaux ». Revue internationale de l'enseignement, juillet à décembre 1899, t. 38, p. 515.

97. - UNIVERSITÉ DE FRANCE. Annuaire des facultés de Bordeaux, 1889-1890. Bordeaux : Vve Cadoret, 1889, p. 123.

98. - UNIVERSITÉ DE BORDEAUX. Livret-guide, 1906-1907. Bordeaux : Vve Cadoret, 1906, p. 93.

99. - UNIVERSITÉ DE BORDEAUX. Livret-guide, 1906-1907. Bordeaux : Vve Cadoret, 1906, p. 93. 
100. - PARIS, Pierre et LA VILLE DE MIRMONT, Henri de. "Le musée de moulages de Bordeaux ». Revue internationale de l'enseignement, juillet à décembre 1899, t. 38, p. 514.

101. - Le récolement des statuettes de Myrina en 2000 comptabilise 101 objets sur les 172 inventoriés initialement. Le récolement des céramiques, des tissus coptes... est à venir.

102. - Le catalogue réalisé pour les photographies ayant disparu, nous ne pouvons nous appuyer pour l'évaluation que sur les chiffres annoncés dans les annuaires des facultés ou dans les textes de Pierre Paris. Malheureusement, ces chiffres sont parfois contradictoires.

103. - MORINIÈRE, Soline. La collection de moulages d'antiques et médiévaux de la Faculté des Lettres de Bordeaux en dépôt au musée d'Aquitaine : étude documentaire, conservation et perspectives. Master 2 professionnel. Paris : École du Louvre, 2011.

104. - RECHT, Roland. "Le moulage et la naissance de l'histoire de l'art». Dans Le musée de sculpture comparée : Naissance de l'histoire de l'art. Paris : Monum-Centre des monuments nationaux, 1999; SCHWARTZ, Emmanuel. "L'anatomie face à l'antique: l'usage du moulage dans l'enseignement académique ». Dans COMAR, Philippe (dir.). Une leçon d'anatomie. Figures du corps à l'École des beaux-Arts. Paris : Beaux-arts de Paris, 2009, p. 83-87.

105. - AMB, délibérations du conseil municipal, 12 D 109, 11 décembre 1894 : les trois salles ouvertes en 1895 concernent les œuvres archaïques qui « demandent une éducation préalable ».

106. - MONDENARD, Anne de. «Des documents d'une apparente objectivité ». Dans Photographier l'architecture 1851-1920. Collection du musée des monuments français. Paris: RMN, 1994, p. 17-31; MATHON, Catherine. "Le modèle photographique ». Dans COMAR, Philippe. Une leçon d'anatomie. Figures du corps à l'École des beaux-Arts. Paris : Beaux-arts de Paris, 2009, p. 111-117.

107. - FREITAG, Wolfgang. "La servante et la séductrice. Histoire de la photographie et histoire de l'art ». Histoire de l'art XVIII et XIX ${ }^{e}$ siècle. Paris : Klinckiek-musée du Louvre, 1997, p. 257-291; THERRIEN, Lyne. L'histoire de l'art en France. Genèse d'une discipline universitaire. Paris : éditions du C.T.H.S., 1998, p. 397-407.

108. - MIANE, Florent. Images d'architectures et imaginaires photographiques, l'œuvre d'Alphonse Terpereau dans le Midi de la France. Doctorat d'histoire de l'art. Bordeaux : Université Michel de Montaigne, 2009, p. 224-231.

109. - L'album des facultés des sciences et des lettres est toujours conservé dans la salle des actes de l'université de Bordeaux. Le musée d'archéologie y apparaît dans trois vues au côté des laboratoires de physique et de chimie.

110. - Une vue de la faculté des lettres et des sciences et un tirage de la lunette méridienne à l'observatoire de Floirac ornent encore certaines salles de l'université.

111. - ARB, conseil général des facultés de Bordeaux, $R$ 23, séance du 28 février 1888 , f 52.

112. - ARB, conseil général des facultés de Bordeaux, $R$ 23, séance du 29 novembre $1892, \mathrm{f}^{\circ} 184$.

113. - Geneviève Dupuis-Sabron, aujourd'hui conservatrice des collections d'histoire contemporaine au musée d'Aquitaine, se souvient avoir suivi des cours de Mme David prenant appui sur les moulages pour étudier le vocabulaire de la sculpture.

\section{RÉSUMÉS}

La fondation du Musée archéologique de la faculté de lettres de Bordeaux en 1886 est la manifestation la plus visible de la nouvelle perception de la sculpture et de l'architecture antiques par les archéologues et, d'une manière générale, de la transformation des 
enseignements universitaires à la fin du XIX siècle. La "redécouverte » de ce musée est l'occasion de revenir sur la genèse des collections universitaires et de livrer une première analyse des fonds aujourd'hui existants.

Founded in 1886, the Archaeological Museum of the Faculty of Letters in Bordeaux is the most visible manifestation of the new perception of sculpture and antique architecture by Archaeologists and, largely, of the transformation of university teaching at the end of the 19th century. The "rediscovery" of this museum is the opportunity to return to the genesis of the university collections and to deliver an initial analysis of existing funds today.

\section{INDEX}

Mots-clés : archéologie, collection, faculté des lettres, histoire de l'histoire de l'art, moulage, musée universitaire, patrimoine, pédagogie, photographie, Université de Bordeaux

\section{AUTEURS}

\section{MARION LAGRANGE}

maître de conférences en histoire de l'art contemporain, porteur du programme Région Patrimoine artistique universitaire de l'université de Bordeaux, Centre de recherches FrançoisGeorges Pariset (E.A. 538), Université Bordeaux 3- Michel de Montaigne

lagrange.marion@gmail.com

\section{FLORENT MIANE}

post-doctorant du programme Région Patrimoine artistique universitaire, Centre de recherches François-Georges Pariset (E.A. 538), Université Bordeaux 3 - Michel de Montaigne

florent.miane@gmail.com 